Article

\title{
Synthesis and Biological Evaluation of Norcantharidin Derivatives Possessing an Aromatic Amine Moiety as Antifungal Agents
}

\author{
Yang Wang ${ }^{1,+}$, Wenbo Sun ${ }^{2,+}$, Shunqing Zha ${ }^{1}$, Huan Wang ${ }^{2}$ and Yalin Zhang ${ }^{2, *}$ \\ Received: 11 November 2015 ; Accepted: 30 November 2015 ; Published: 2 December 2015 \\ Academic Editor: Derek J. McPhee \\ 1 State Key Laboratory of Crop Stress Biology for Arid Areas, College of Plant Protection, Northwest A \& F \\ University, Yangling 712100, China; wangyang2006@nwsuaf.edu.cn (Y.W.); zsq2623@163.com (S.Z.) \\ 2 Key Laboratory of Plant Protection Resources and Pest Management, Ministry of Education, \\ Northwest A \& F University, Yangling 712100, China; wbsun06@163.com (W.S.); \\ huanwang1107@gmail.com (H.W.) \\ * Correspondence: yalinzh@nwsuaf.edu.cn; Tel./Fax: +86-29-8709-2190 \\ + These authors contributed equally to this work.
}

\begin{abstract}
Based on the structure of naturally produced cantharidin, different arylamine groups were linked to the norcantharidin scaffold to provide thirty six compounds. Their structures were confirmed by melting point, ${ }^{1} \mathrm{H}-\mathrm{NMR},{ }^{13} \mathrm{C}-\mathrm{NMR}$ and HRMS-ESI studies. These synthetic compounds were tested as fungistatic agents against eight phytopathogenic fungi using the mycelium growth rate method. Of these thirty six derivatives, seven displayed stronger antifungal activity than did norcantharidin, seven showed higher activity than did cantharidin and three exhibited more significant activity than that of thiabendazole. In particular, 3-(3'-chloro-phenyl)carbamoyl norcantharidate II-8 showed the most significant fungicidal activity against Sclerotinia fructigena and S. sclerotiorum, with $\mathrm{IC}_{50}$ values of 0.88 and $0.97 \mu \mathrm{g} / \mathrm{mL}$, respectively. The preliminary structure-activity relationship data of these compounds revealed that: (1) the benzene ring is critical for the improvement of the spectrum of antifungal activity (3-phenylcarbamoyl norcantharidate II-1 vs norcantharidin and cantharidin); (2) among the three sites, including the $\mathrm{C}-2^{\prime}, \mathrm{C}-3^{\prime}$ and $\mathrm{C}-4^{\prime}$ positions of the phenyl ring, the presence of a halogen atom at the $\mathrm{C}-3^{\prime}$ position of the benzene ring caused the most significant increase in antifungal activity; (3) compounds with strongly electron-drawing or electron-donating groups substitutions were found to have a poor antifungal activity; and (4) compared with fluorine, bromine and iodine, chlorine substituted at the $\mathrm{C}-3^{\prime}$ position of the benzene ring most greatly promoted fungistatic activity. Thus, compound II-8 has emerged as new lead structure for the development of new fungicides.
\end{abstract}

Keywords: cantharidin; natural product; structural modification; arylamine; fungicidal activity

\section{Introduction}

Fungal pathogens are the primary causes of both plant diseases and postharvest losses [1,2], and thus contribute to severe damage to global crop production [3]. To guard against fungal pathogens, one traditional approach is to employ synthetic fungicides, which are both economical and efficient, and have played an indispensable role in nourishing more people throughout human history. Unfortunately, drug resistance, environmental hazards and many other drawbacks have emerged along with this fungicide utilization $[4,5]$. This requires that novel antifungal agents continue to be discovered. It is well-known that insect secondary metabolites result from their adaptation to the environments during the long period of evolution in insects, such as for defense against predation 
or infection, and pesticides produced from insect secondary metabolites may result in less or slower resistance development and lower pollution [6]. Hence, insecticides of natural source have been considered as attractive alternatives to synthetic agrochemicals for pest management [7]. Bio-based insecticides, such as nereistoxin, nicotine, pyrethrum, and neem extracts, are made by organisms as defense against insects [8].

Cantharidin (CTD, Figure 1A), a naturally occurring terpene, is the main secondary metabolite isolated from the bodies of the blister beetles, including Mylabris cichorii, M. phalerata and Epicauta chinensis $[9,10]$. Besides its use as the lead compound for the preparation of potent anticancer drugs, such as its analogues sodium cantharidinate and norcantharidin [11-14], CTD also shows interesting antifungal and insecticidal activities [15-18].

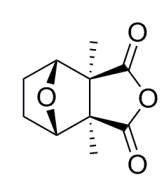

A

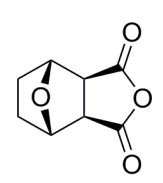

B

Figure 1. The structures of cantharidin (A) and norcantharidin (B).

Norcantharidin (NCTD, Figure 1B), a demethylated analogue of CTD, is well known as a strong inhibitor of protein serine/threonine phosphatases (PSPs) [19], a broad class of PSPs associated with signaling and control of numerous cellular processes in many organisms [20]. The biological activities associated with NCTD are derived from its abilities to inhibit the family of PSPs. Furthermore, the catalytic domain of all PSP subfamilies is highly conserved in insects, plants, phytopathogenic fungi and all the other eukaryotes [21]. NCTD, as the strong inhibitor, binds to a hydrophobic pocket of the PSP active site [22]. The structural similarity between CTD and NCTD has been apparent to animal scientists and similar mechanisms of action on animals have been confirmed [23]. Previous studies by our group demonstrated that both in vivo and in vitro, there are significant inhibitory effects from CTD, NCTD and their analogues on PSPs of Plutella xylostella, suggesting that their modes of action may be related to impeding insect PSPs activity [24]. More recently, strong evidence indicates that the commercial herbicide mode of action of endothall, an analogue of NCTD, was intimately related to the inhibition of PSPs [25].

Meanwhile, as illustrated in Figure 2, our previous investigation on the structure-activity relationship (SAR) of NCTD and its anhydride modified derivatives against $P$. xylostella indicated that the structures of oxygen bridge and carboxyl were essential for the biological activity, and that the improvement of bioactivity required a reasonable $\mathrm{R}$ group, the combination of both aliphatic amide and aromatic amide moieties. And the type of substituent $Y$, substituted on the phenyl ring, was also critical for the improvement of insecticidal activity [26].

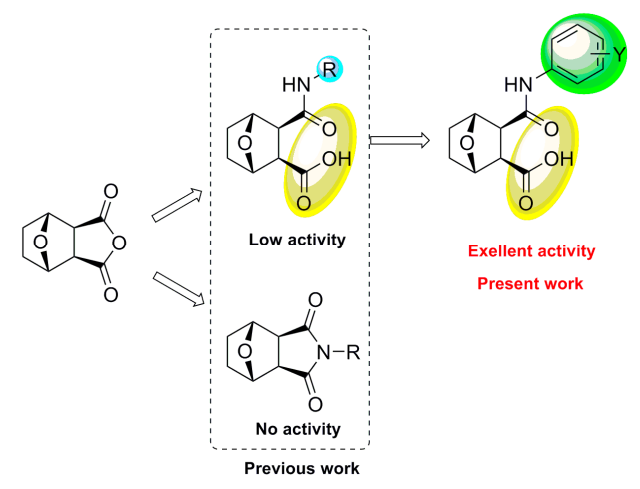

Figure 2. Design of the title compounds. 
To date, few attempts have been made to develop a biorational fungicide from NCTD via chemical modification. Encouraged by the abovementioned results and in continuation of our program aimed at the discovery and development of natural-product-based fungicidal agents, in this study, thirty six aromatic amine derivatives of NCTD modified in the anhydride ring were synthesized (Scheme 1), and their biological activities against eight phytopathogenic fungi were evaluated using the mycelium growth rate method [27]. Additionally, their preliminary SAR studies were also described.

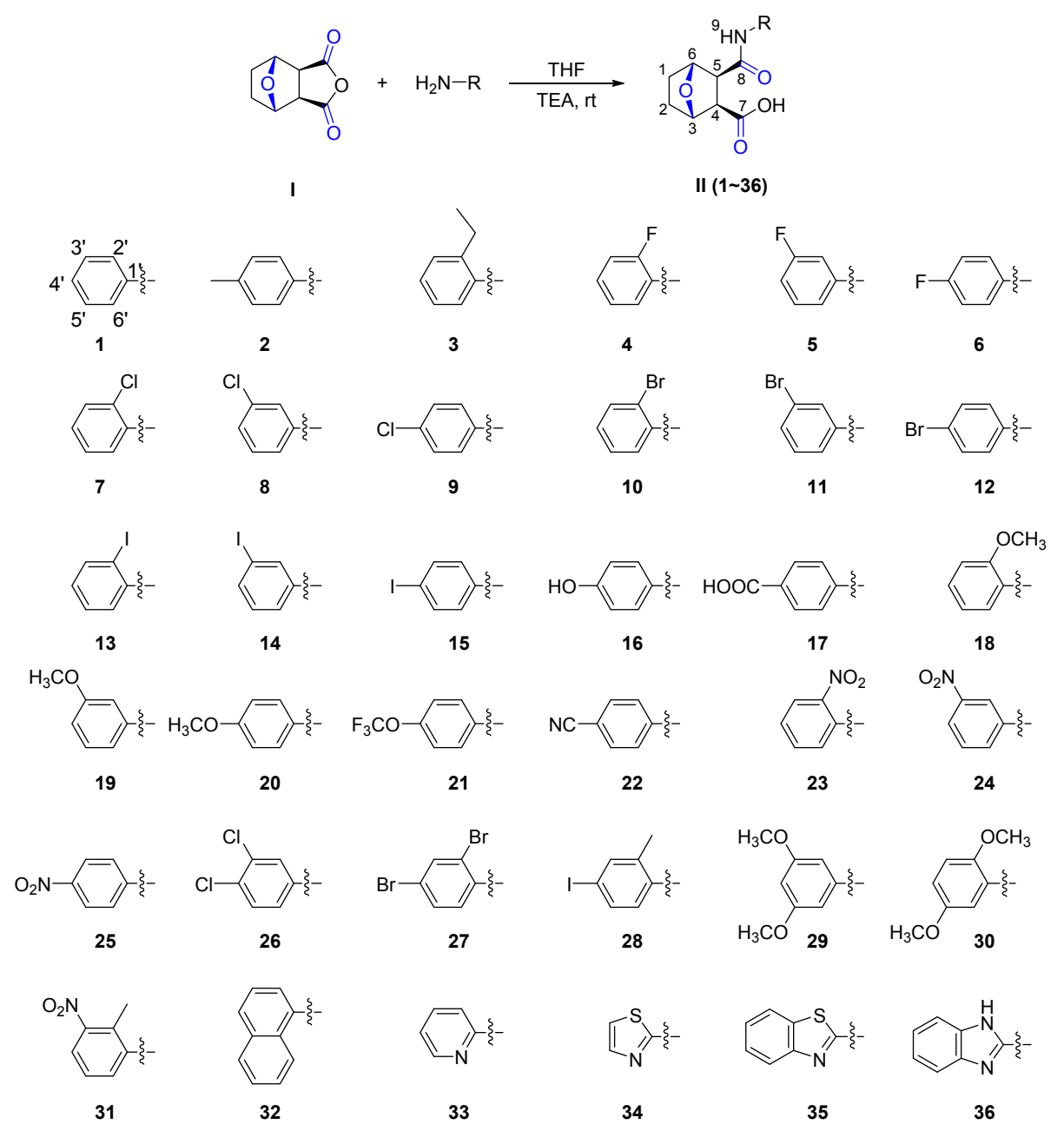

Scheme 1. The Synthesis and Structures of Compounds II (1-36).

\section{Results and Discussion}

\subsection{Chemistry}

As shown in Scheme 1, the target compounds II were synthesized by the aminolysis reaction of NCTD I and various halogen aromatic amines in the presence of triethylamine as the binding acid agent, and synthesized via decomposing the anhydride ring of NCTD I by a halogenated aniline with different electronegativity [28].

\subsection{Antifungal Activity}

Preliminary in vitro screening results of the title compounds for antifungal activities against eight fungi at the concentration of $50 \mu \mathrm{g} / \mathrm{mL}$ are listed in Table 1. 
Table 1. Preliminary antifungal activities of compounds at $50 \mu \mathrm{g} / \mathrm{mL}$.

\begin{tabular}{|c|c|c|c|c|c|c|c|c|}
\hline \multirow[b]{2}{*}{ Compd. } & \multicolumn{8}{|c|}{ Values of Inhibition Rate (\%) to Eight Pathogens } \\
\hline & Valsa mali & $\begin{array}{c}\text { Botryosphaeria } \\
\text { berengeriana }\end{array}$ & $\begin{array}{l}\text { Sclerotinia } \\
\text { fructigena }\end{array}$ & $\begin{array}{l}\text { Glomerella } \\
\text { cingulate }\end{array}$ & $\begin{array}{l}\text { Alternaria } \\
\text { alternate }\end{array}$ & $\begin{array}{c}\text { Sclerotinia } \\
\text { sclerotiorum }\end{array}$ & Alternaria solani & $\begin{array}{c}\text { Cochliobolus } \\
\text { sativum }\end{array}$ \\
\hline II-1 & $68.42 \pm 1.99 \mathrm{~d}^{*}$ & $68.64 \pm 0.65 \mathrm{e}$ & $72.20 \pm 0.66 \mathrm{e}$ & $62.35 \pm 0.99 f$ & $65.52 \pm 1.06 \mathrm{de}$ & $76.39 \pm 0.92 \mathrm{e}$ & $61.10 \pm 0.96 \mathrm{e}$ & $67.01 \pm 0.59 c$ \\
\hline II-2 & $0.66 \pm 0.06 q$ & $1.27 \pm 0.03 q \mathrm{qr}$ & $0.53 \pm 0.06 \mathrm{qr}$ & $5.38 \pm 0.05 \mathrm{tu}$ & $9.66 \pm 0.06$ no & $23.61 \pm 0.06 p$ & $1.52 \pm 0.10 \mathrm{u}$ & $7.98 \pm 0.06 \mathrm{qr}$ \\
\hline II-3 & $18.42 \pm 0.06 \mathrm{mn}$ & $11.02 \pm 0.101$ & $18.72 \pm 0.06 \mathrm{~m}$ & $6.99 \pm 0.06 \mathrm{st}$ & $11.72 \pm 0.06 n$ & $20.17 \pm 0.00 \mathrm{q}$ & $1.01 \pm 0.06 \mathrm{u}$ & $10.11 \pm 0.06 \mathrm{pqr}$ \\
\hline II-4 & $74.99 \pm 1.30 c$ & $69.90 \pm 1.17 \mathrm{e}$ & $76.48 \pm 1.63 \mathrm{~d}$ & $80.66 \pm 1.18 \mathrm{~d}$ & $70.34 \pm 1.41 c$ & $86.69 \pm 0.85 \mathrm{~d}$ & $76.77 \pm 0.26 c$ & $66.50 \pm 0.30 c$ \\
\hline II-5 & $83.56 \pm 1.05 b$ & $87.70 \pm 0.91 c$ & $85.03 \pm 1.79 b c$ & $90.34 \pm 2.64 b$ & $84.82 \pm 1.30 \mathrm{a}$ & $92.27 \pm 0.06 c$ & $83.35 \pm 1.16 b$ & $81.39 \pm 0.38 b$ \\
\hline II-6 & $41.45 \pm 0.47 \mathrm{e}$ & $39.39 \pm 1.61 \mathrm{f}$ & $49.21 \pm 1.37 f$ & $55.40 \pm 1.36 \mathrm{~g}$ & $41.38 \pm 2.14 \mathrm{~g}$ & $46.34 \pm 2.29 f$ & $47.46 \pm 1.21 \mathrm{f}$ & $51.59 \pm 0.76 \mathrm{e}$ \\
\hline II-7 & $34.21 \pm 1.00 \mathrm{f}$ & $22.86 \pm 1.85 h$ & $45.47 \pm 2.26 \mathrm{~g}$ & $24.74 \pm 0.83 \mathrm{~h}$ & $38.62 \pm 1.04 \mathrm{~g}$ & $35.19 \pm 1.78 \mathrm{~g}$ & $30.29 \pm 0.92 \mathrm{~g}$ & $35.06 \pm 1.80 \mathrm{f}$ \\
\hline II-8 & $86.85 \pm 1.00 \mathrm{a}$ & $91.54 \pm 1.33 b$ & $100.00 \pm 0.00 \mathrm{a}$ & $92.96 \pm 2.69 a$ & $86.90 \pm 2.32 a$ & $100.00 \pm 0.00 \mathrm{a}$ & $89.90 \pm 0.79 a$ & $91.50 \pm 0.66 a$ \\
\hline II-9 & $32.88 \pm 3.85 f$ & $22.01 \pm 2.89 h$ & $28.36 \pm 2.69 h$ & $24.18 \pm 1.08 \mathrm{~h}$ & $30.34 \pm 3.13 h$ & $26.60 \pm 2.79 \mathrm{i}$ & $28.27 \pm 1.83 \mathrm{hi}$ & $17.53 \pm 0.90 \mathrm{j}$ \\
\hline II-10 & $25.01 \pm 3.06 \mathrm{~g}$ & $18.18 \pm 3.79 \mathrm{i}$ & $23.00 \pm 2.51 \mathrm{i}$ & $21.48 \pm 1.28 \mathrm{i}$ & $23.44 \pm 2.27 \mathrm{i}$ & $30.46 \pm 3.04 \mathrm{~h}$ & $18.17 \pm 2.45 \mathrm{j}$ & $21.21 \pm 2.53 \mathrm{i}$ \\
\hline II-11 & $80.92 \pm 1.26 b$ & $81.77 \pm 0.90 \mathrm{~d}$ & $83.42 \pm 2.49 c$ & $84.97 \pm 1.47 c$ & $78.63 \pm 2.28 b$ & $96.13 \pm 1.32 b$ & $79.83 \pm 1.81 b c$ & $83.56 \pm 1.80 b$ \\
\hline II-12 & $20.39 \pm 1.04 \mathrm{~h}$ & $15.24 \pm 3.23 i$ & $17.13 \pm 2.59 j$ & $18.25 \pm 1.37 \mathrm{j}$ & $22.07 \pm 3.14 \mathrm{i}$ & $20.59 \pm 2.45 \mathrm{j}$ & $25.23 \pm 1.87 \mathrm{i}$ & $13.78 \pm 1.91 \mathrm{k}$ \\
\hline II-13 & $15.14 \pm 1.23 \mathrm{i}$ & $10.99 \pm 2.47 j$ & $22.46 \pm 1.62 \mathrm{i}$ & $12.35 \pm 0.59 \mathrm{k}$ & $17.22 \pm 4.17 j$ & $15.87 \pm 1.37 \mathrm{k}$ & $16.16 \pm 2.31 j$ & $9.54 \pm 1.221$ \\
\hline II-14 & $65.79 \pm 1.00 \mathrm{~d}$ & $66.51 \pm 1.95 \mathrm{e}$ & $70.60 \pm 2.19 \mathrm{e}$ & $60.77 \pm 0.78 f$ & $62.76 \pm 2.13 \mathrm{e}$ & $73.80 \pm 3.43 \mathrm{e}$ & $58.60 \pm 1.33 \mathrm{e}$ & $63.86 \pm 1.01 \mathrm{~d}$ \\
\hline II-15 & $3.29 \pm 1.16 \mathrm{j}$ & $-5.12 \pm 3.86 \mathrm{k}$ & $1.07 \pm 0.92 \mathrm{k}$ & $5.37 \pm 0.861$ & $3.44 \pm 1.18 \mathrm{k}$ & $8.15 \pm 1.431$ & $11.10 \pm 6.18 \mathrm{k}$ & $-2.71 \pm 1.98 m$ \\
\hline II-16 & $9.21 \pm 0.10 p$ & $0.85 \pm 0.04 \mathrm{pqr}$ & $8.56 \pm 0.06 p$ & $9.14 \pm 0.03 \mathrm{rs}$ & $10.34 \pm 0.06 \mathrm{no}$ & $6.87 \pm 0.06 t$ & $2.02 \pm 0.10 \mathrm{u}$ & $5.85 \pm 0.10 \mathrm{~s}$ \\
\hline II-17 & $0.66 \pm 0.06 q$ & $0.42 \pm 0.06 \mathrm{qr}$ & $-0.53 \pm 0.06 \mathrm{r}$ & $0.54 \pm 0.06 w x$ & $1.38 \pm 0.06 \mathrm{pq}$ & $0.43 \pm 0.08 u$ & $7.58 \pm 0.12 \mathrm{~s}$ & $12.23 \pm 0.10$ nopq \\
\hline II-18 & $1.97 \pm 0.06 q$ & $6.36 \pm 0.06 \mathrm{mn}$ & $0.00 \pm 0.00 \mathrm{qr}$ & $0.00 \pm 0.00 x$ & $8.28 \pm 0.06$ no & $0.00 \pm 0.06 \mathrm{u}$ & $0.00 \pm 0.00 \mathrm{u}$ & $0.00 \pm 0.00 \mathrm{t}$ \\
\hline II-19 & $0.66 \pm 0.06 q$ & $10.1 \overline{7} \pm 0.061$ & $2.67 \pm 0.06 \mathrm{qr}$ & $11.83 \pm 0.05 \mathrm{pq}$ & $15.86 \pm 0.06 \mathrm{~m}$ & $0.00 \pm 0.06 \mathrm{u}$ & $2.53 \pm 0.00 \mathrm{u}$ & $11.70 \pm 0.06 \mathrm{opq}$ \\
\hline II-20 & $0.66 \pm 0.06 q$ & $2.12 \pm 0.10 \mathrm{pqr}$ & $0.53 \pm 0.06 q r$ & $0.00 \pm 0.00 \mathrm{x}$ & $2.07 \pm 0.06 \mathrm{pq}$ & $0.00 \pm 0.06 \mathrm{u}$ & $0.00 \pm 0.00 \mathrm{u}$ & $0.00 \pm 0.00 \mathrm{t}$ \\
\hline II-21 & $1.32 \pm 0.10 \mathrm{q}$ & $10.59 \pm 0.061$ & $11.76 \pm 0.10 \mathrm{o}$ & $15.59 \pm 0.06 o$ & $20.69 \pm 0.06 \mathrm{ijk}$ & $13.30 \pm 0.06 \mathrm{~s}$ & $21.72 \pm 0.061 \mathrm{~m}$ & $22.87 \pm 0.06 \mathrm{hi}$ \\
\hline II-22 & $24.34 \pm 0.06 \mathrm{k}$ & $0.00 \pm 0.066 \mathrm{r}$ & $8.02 \pm 0.11 p$ & $12.90 \pm 0.05 p$ & $17.93 \pm 0.06 \mathrm{jklm}$ & $18.45 \pm 0.06 q$ & $22.73 \pm 0.06 \mathrm{kl}$ & $12.23 \pm 0.03$ nopq \\
\hline II-23 & $19.74 \pm 0.06 \mathrm{~m}$ & $0.00 \pm 0.00 \mathrm{r}$ & $9.09 \pm 0.11 p$ & $3.23 \pm 0.03 u v$ & $0.69 \pm 0.10 \mathrm{pq}$ & $13.73 \pm 0.10 \mathrm{t}$ & $2.53 \pm 0.03 u$ & $11.17 \pm 0.06 \mathrm{opq}$ \\
\hline III-24 & $25.00 \pm 0.10 q$ & $5.08 \pm 0.06 n$ & $18.18 \pm 0.10 \mathrm{~m}$ & $20.43 \pm 0.06 \mathrm{~m}$ & $28.28 \pm 0.06 h$ & $19.31 \pm 0.06 q$ & $7.07 \pm 0.03 \mathrm{st}$ & $15.43 \pm 0.10 \mathrm{~lm}$ \\
\hline II-25 & $23.03 \pm 0.10 \mathrm{kl}$ & $2.54 \pm 0.11 p q$ & $12.30 \pm 0.060$ & $17.20 \pm 0.05 \mathrm{no}$ & $21.38 \pm 0.10 \mathrm{ij}$ & $7.73 \pm 0.06 u$ & $3.54 \pm 0.03 \mathrm{tu}$ & $6.38 \pm 0.11 \mathrm{rs}$ \\
\hline II-26 & $32.89 \pm 0.10 \mathrm{j}$ & $18.22 \pm 0.06 \mathrm{k}$ & $48.13 \pm 0.06 \mathrm{~g}$ & $28.49 \pm 0.06 \mathrm{~h}$ & $35.86 \pm 0.10 \mathrm{~g}$ & $40.34 \pm 0.06 \mathrm{k}$ & $33.33 \pm 0.12 o p$ & $37.23 \pm 0.06 f$ \\
\hline II-27 & $36.18 \pm 0.06 \mathrm{hi}$ & $15.25 \pm 0.06 \mathrm{j}$ & $39.57 \pm 0.06 \mathrm{i}$ & $22.04 \pm 0.06 \mathrm{jkl}$ & $20.69 \pm 0.06 \mathrm{ijk}$ & $42.96 \pm 0.06 \mathrm{j}$ & $29.80 \pm 0.06 \mathrm{ij}$ & $14.89 \pm 0.061 \mathrm{mn}$ \\
\hline II-28 & $8.55 \pm 0.06 p$ & $6.78 \pm 0.060$ & $39.04 \pm 0.06 \mathrm{i}$ & $23.66 \pm 0.06 \mathrm{ijk}$ & $38.62 \pm 0.10 \mathrm{fg}$ & $42.06 \pm 0.00 \mathrm{jk}$ & $16.67 \pm 0.10$ nop & $12.77 \pm 0.06 \mathrm{mnop}$ \\
\hline II-29 & $30.26 \pm 0.06 j$ & $0.42 \pm 0.06 \mathrm{qr}$ & $36.36 \pm 0.06 j$ & $21.51 \pm 0.06 \mathrm{kl}$ & $40.00 \pm 0.06 f$ & $36.48 \pm 0.061$ & $20.20 \pm 0.061 \mathrm{mn}$ & $12.77 \pm 0.10$ mnop \\
\hline II-30 & $0.66 \pm 0.06 q$ & $0.00 \pm 0.06 \mathrm{r}$ & $14.97 \pm 0.10 \mathrm{n}$ & $4.30 \pm 0.06 \mathrm{tuv}$ & $10.34 \pm 0.16$ no & $26.18 \pm 0.06 \mathrm{no}$ & $12.12 \pm 0.10 \mathrm{qr}$ & $18.62 \pm 0.10 \mathrm{jk}$ \\
\hline II-31 & $15.13 \pm 0.10 \mathrm{o}$ & $0.00 \pm 0.06 \mathrm{r}$ & $17.11 \pm 0.06 \mathrm{~m}$ & $4.84 \pm 0.00$ tuv & $-1.38 \pm 0.06 q$ & $12.02 \pm 0.06 \mathrm{~s}$ & $7.07 \pm 0.10 \mathrm{st}$ & $12.23 \pm 0.11$ nopq \\
\hline II-32 & $3.29 \pm 0.10 \mathrm{q}$ & $8.05 \pm 0.06 \mathrm{~m}$ & $6.42 \pm 0.06 p$ & $5.91 \pm 0.06 \mathrm{tu}$ & $6.90 \pm 0.060$ & $7.30 \pm 0.00 \mathrm{t}$ & $18.69 \pm 0.06 \mathrm{mno}$ & $22.34 \pm 0.06 h i$ \\
\hline II-33 & $0.66 \pm 0.06 q$ & $0.00 \pm 0.11 \mathrm{r}$ & $28.88 \pm 0.06 \mathrm{k}$ & $2.69 \pm 0.06 \mathrm{vw}$ & $-0.69 \pm 0.05 q$ & $23.61 \pm 0.08 p$ & $6.57 \pm 0.06 \mathrm{st}$ & $-1.60 \pm 0.07 t$ \\
\hline
\end{tabular}


Table 1. Cont.

\begin{tabular}{|c|c|c|c|c|c|c|c|c|}
\hline \multirow[b]{2}{*}{ Compd. } & \multicolumn{8}{|c|}{ Values of Inhibition Rate (\%) to Eight Pathogens } \\
\hline & Valsa mali & $\begin{array}{c}\text { Botryosphaeria } \\
\text { berengeriana }\end{array}$ & $\begin{array}{l}\text { Sclerotinia } \\
\text { fructigena }\end{array}$ & $\begin{array}{l}\text { Glomerella } \\
\text { cingulate }\end{array}$ & $\begin{array}{l}\text { Alternaria } \\
\text { alternate }\end{array}$ & $\begin{array}{c}\text { Sclerotinia } \\
\text { sclerotiorum }\end{array}$ & $\begin{array}{l}\text { Alternaria } \\
\text { solani }\end{array}$ & $\begin{array}{l}\text { Cochliobolus } \\
\text { sativum }\end{array}$ \\
\hline II-34 & $36.84 \pm 0.10 h$ & $0.42 \pm 0.06 \mathrm{qr}$ & $30.48 \pm 0.06 \mathrm{k}$ & $0.54 \pm 0.06 x$ & $0.69 \pm 0.06 \mathrm{pq}$ & $69.53 \pm 0.06 h$ & $13.13 \pm 0.06 \mathrm{pqr}$ & $15.43 \pm 0.101 \mathrm{~m}$ \\
\hline II-35 & $30.26 \pm 0.15 g$ & $2.97 \pm 0.060$ & $21.39 \pm 0.101$ & $5.91 \pm 0.06 \mathrm{tu}$ & $0.00 \pm 0.03 p q$ & $0.00 \pm 0.00 \mathrm{u}$ & $0.51 \pm 0.06 \mathrm{u}$ & $10.11 \pm 0.03 \mathrm{pqr}$ \\
\hline II-36 & $61.93 \pm 0.10 \mathrm{e}$ & $72.88 \pm 0.06 \mathrm{e}$ & $68.98 \pm 0.15 f$ & $62.37 \pm 0.06 \mathrm{e}$ & $61.38 \pm 0.03 \mathrm{~d}$ & $77.25 \pm 0.06 f$ & $62.63 \pm 0.06 \mathrm{e}$ & $65.43 \pm 0.03 \mathrm{~cd}$ \\
\hline Norcantharidin & $88.17 \pm 1.87 \mathrm{a}$ & $34.31 \pm 1.69 \mathrm{~g}$ & $86.64 \pm 0.80 \mathrm{~b}$ & $17.72 \pm 1.21 \mathrm{j}$ & $20.00 \pm 1.10 \mathrm{ij}$ & $24.46 \pm 1.13 \mathrm{i}$ & $1.98 \pm 2.241$ & $29.75 \pm 1.27 \mathrm{~g}$ \\
\hline Cantharidin & $88.16 \pm 1.39 \mathrm{a}$ & $38.54 \pm 2.68 \mathrm{f}$ & $100.00 \pm 0.00 \mathrm{a}$ & $10.19 \pm 1.61 \mathrm{k}$ & $67.59 \pm 1.06 \mathrm{~cd}$ & $84.54 \pm 1.39 \mathrm{~d}$ & $70.20 \pm 0.84 d$ & $68.05 \pm 1.29 c$ \\
\hline Thiabendazole & $86.84 \pm 1.22 \mathrm{a}$ & $100.00 \pm 0.00 \mathrm{a}$ & $100.0 \pm 0.00 \mathrm{a}$ & $66.67 \pm 0.00 \mathrm{e}$ & $47.58 \pm 1.60 \mathrm{f}$ & $100.00 \pm 0.00 \mathrm{a}$ & $15.14 \pm 1.18 \mathrm{j}$ & $24.99 \pm 1.17 \mathrm{~h}$ \\
\hline
\end{tabular}

* The differences between data with different letters within a column are significant for the same tested fungus $(p<0.05)$ with respect to TBZ, cantharidin, norcantharidin and these synthetic compounds with a halogenated benzene moiety. 
The results in Table 1 indicate that seven of the synthetic derivatives displayed significant activities $(61.1 \%-100 \%$ inhibitory rate) against all eight tested fungi at a concentration of $50 \mu \mathrm{g} / \mathrm{mL}$. On the contrary, another twenty nine derivatives showed lower activity at the same concentration. Here, the inhibition rates were for only given as $\mathrm{IC}_{50}$ values for further comparison.

Encouraged by these preliminary findings, we planned further SAR studies on the title compounds. We determined their $\mathrm{IC}_{50}$ values by the mycelial growth inhibitory rate method. As shown in Table 2, the tested compounds presented different fungicidal activity against the eight plant pathogenic fungi, superior to the corresponding parent compound NCTD in some cases, and they were the same as or more active than thiabendazole (TBZ) against some of the tested fungi. Of these compounds, 3-(3'-chlorophenyl)carbamoyl norcantharidate II-8 exhibited the most significant activity on all the eight fungi. As illustrated in Figure 3, compound II-8 showed much better activity than that of TBZ, NCTD and CTD. Notably, compound II-8 showed excellent antifungal properties against Sclerotinia fructigena and S. sclerotiorum, with $\mathrm{IC}_{50}$ values of 0.88 and $0.97 \mu \mathrm{g} / \mathrm{mL}$, respectively.

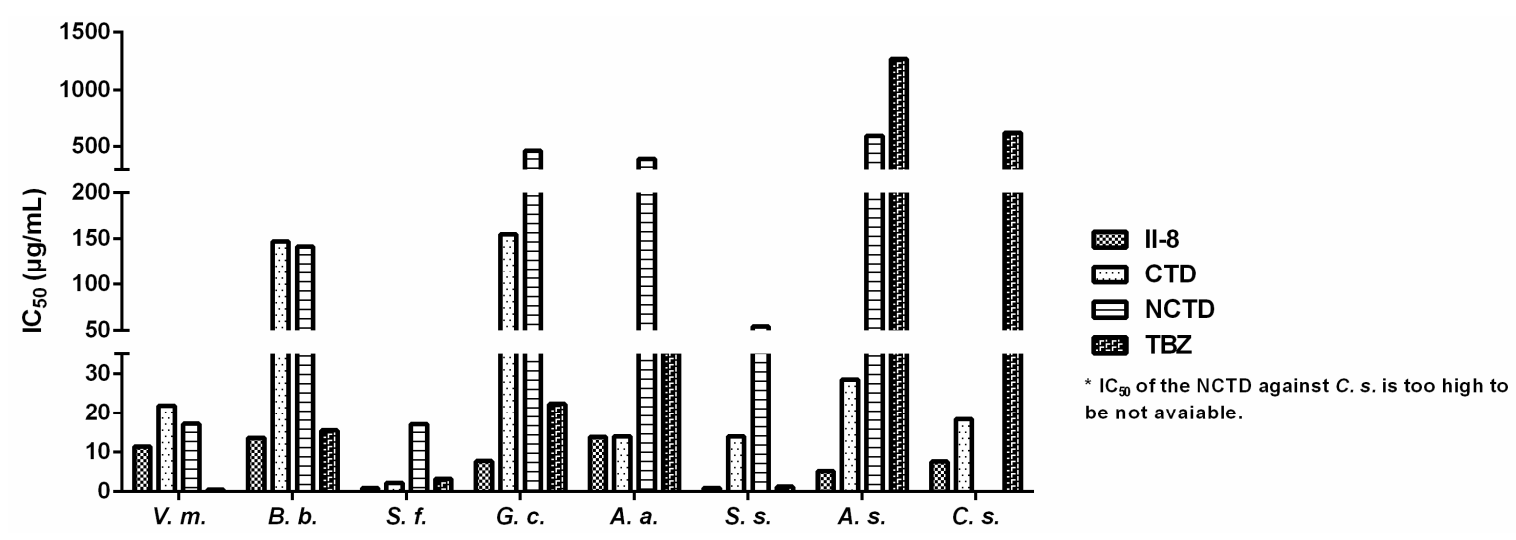

Figure 3. IC $_{50}$ of compound II-8, CTD, NCTD and TBZ against the eight tested fungi.

\section{3. $S A R$}

\subsubsection{Effect of Introducing the Benzene Ring on Fungistatic Activity}

Illustrated in Table 2, NCTD, without a benzene ring, exhibited low fungistatic activity at the concentration of $50 \mu \mathrm{g} / \mathrm{mL}$ against the tested strains. Introducing one benzene (2a) caused a significant increase in fungistatic activity against the plant-pathogenic fungi Botryosphaeria berengeriana, S. fructigena, Glomerella cingulate, Alternaria alternate, S. sclerotiorum, A. solani and Cochliobolus sativum, with $\mathrm{IC}_{50}$ values of 31.2568, 14.6778, 36.3526, 42.2537, 7.2482, 30.1335 and $22.7788 \mu \mathrm{g} / \mathrm{mL}$, respectively. At the same time, as seen in Table 2, compared with the fungicidal activities of CTD against B. berengeriana and G. cingulate, the compound II-1 also showed almost 5 -fold more fungistatic activity than CTD, respectively. On the contrary, the introduction of a heterocyclic ring gave us a negative contribution to fungistatic activity.

\subsubsection{Effect of Position Substituted on the Benzene Ring on Fungistatic Activity}

Substituting a halogen at the C-3'position of the benzene ring (i.e., compounds II-5, II-8 and II-11) improved the fungicidal activity in some cases. It was also clear that the site substituted on the phenyl ring plays an important role in activity, as compounds substituted at the C- $4^{\prime}$ position (II-6, II-9 and II-12) and C-2' position (II-4, II-7 and II-10) were all found to be less active than their analogues. 
Table 2. Antifungal activity of some compounds against eight pathogens *

\begin{tabular}{|c|c|c|c|c|c|c|c|c|}
\hline \multirow{2}{*}{ Compd. } & \multicolumn{8}{|c|}{$\operatorname{IC50}(\mu \mathrm{g} / \mathrm{mL})(\mathrm{CI} 95 \%) * *$} \\
\hline & $V . m$. & B. $b$. & S. $f$. & G.c. & A. $a$. & S. s. & A. $s$. & C.s. \\
\hline II-1 & $\begin{array}{c}32.0800 \\
(26.1510-39.3533)\end{array}$ & $\begin{array}{c}31.2568 \\
(23.7127-41.2012)\end{array}$ & $\begin{array}{c}14.6778 \\
(10.7872-19.9716)\end{array}$ & $\begin{array}{c}36.3526 \\
(29.1302-45.3658)\end{array}$ & $\begin{array}{c}42.2537 \\
(33.7577-52.8879)\end{array}$ & $\begin{array}{c}7.2482 \\
(2.9912-17.5637)\end{array}$ & $\begin{array}{c}30.1335 \\
(23.3387-38.9065)\end{array}$ & $\begin{array}{c}22.7788 \\
(16.0894-32.2493)\end{array}$ \\
\hline II-4 & $\begin{array}{c}27.8016 \\
(22.1312-34.9249)\end{array}$ & $\begin{array}{c}24.8446 \\
(18.2585-33.8063)\end{array}$ & $\begin{array}{c}10.6665 \\
(7.6148-14.9412)\end{array}$ & $\begin{array}{c}16.7764 \\
(11.7587-23.9352)\end{array}$ & $\begin{array}{c}31.4258 \\
(24.6239-40.1066)\end{array}$ & $\begin{array}{c}4.2858 \\
(1.6648-11.0334)\end{array}$ & $\begin{array}{c}14.0938 \\
(9.1043-21.8178)\end{array}$ & $\begin{array}{c}10.4051 \\
(12.9130-24.7109)\end{array}$ \\
\hline II-5 & $\begin{array}{c}20.1058 \\
(14.8666-27.1914)\end{array}$ & $\begin{array}{c}19.8375 \\
(14.7821-26.6217)\end{array}$ & $\begin{array}{c}2.0791 \\
(1.3098-3.3003)\end{array}$ & $\begin{array}{c}11.2505 \\
(6.9283-18.2691)\end{array}$ & $\begin{array}{c}18.1002 \\
(13.1268-24.9579)\end{array}$ & $\begin{array}{c}3.4556 \\
(1.6958-7.0418)\end{array}$ & $\begin{array}{c}10.4753 \\
(6.7490-16.2590)\end{array}$ & $\begin{array}{c}10.4051 \\
(6.4109-16.8880)\end{array}$ \\
\hline II-8 & $\begin{array}{c}11.3756 \\
(7.2790-17.7778)\end{array}$ & $\begin{array}{c}13.6528 \\
(9.8721-18.8814)\end{array}$ & $\begin{array}{c}0.8805 \\
(0.4243-1.8275)\end{array}$ & $\begin{array}{c}7.7364 \\
(3.8335-15.6128)\end{array}$ & $\begin{array}{c}13.8916 \\
(9.3377-20.6664)\end{array}$ & $\begin{array}{c}0.9698 \\
(0.4790-1.9633)\end{array}$ & $\begin{array}{c}5.1863 \\
(2.5133-10.7020)\end{array}$ & $\begin{array}{c}7.5908 \\
(4.4564-12.9298)\end{array}$ \\
\hline II-11 & $\begin{array}{c}17.0896 \\
(11.9965-23.3450) \\
\end{array}$ & $\begin{array}{c}17.0640 \\
(12.6223-23.0689) \\
\end{array}$ & $\begin{array}{c}2.3717 \\
(1.4367-3.9152) \\
\end{array}$ & $\begin{array}{c}9.1071 \\
(5.1797-16.0125) \\
\end{array}$ & $\begin{array}{c}22.1465 \\
(16.7792-29.2308) \\
\end{array}$ & $\begin{array}{c}2.1783 \\
(0.9142-5.1904) \\
\end{array}$ & $\begin{array}{c}12.1081 \\
(7.9591-18.4200) \\
\end{array}$ & $\begin{array}{c}12.9298 \\
(8.9552-18.6685) \\
\end{array}$ \\
\hline II-14 & $\begin{array}{c}47.7810 \\
(39.0992-58.39 .4)\end{array}$ & $\begin{array}{c}37.3895 \\
(28.8394-48.4746)\end{array}$ & $\begin{array}{c}21.9666 \\
(16.3757-29.4664)\end{array}$ & $\begin{array}{c}57.0963 \\
(45.2452-72.0517)\end{array}$ & $\begin{array}{c}49.1546 \\
(39.1730-61.6795)\end{array}$ & $\begin{array}{c}9.6192 \\
(4.2886-21.5754)\end{array}$ & $\begin{array}{c}34.8290 \\
(27.5093-44.0964)\end{array}$ & $\begin{array}{c}29.8409 \\
(16.0894-32.2493)\end{array}$ \\
\hline Norcantharidin & $\begin{array}{c}17.1862 \\
(9.2272-32.0104)\end{array}$ & $\begin{array}{c}141.8133 \\
(61.1287-328.9946)\end{array}$ & $\begin{array}{c}17.1673 \\
(10.0952-29.1937)\end{array}$ & $\begin{array}{c}465.1719 \\
(86.5242-2500.8598)\end{array}$ & $\begin{array}{c}394.9566 \\
(73.9654-2108.9689)\end{array}$ & $\begin{array}{c}54.5899 \\
(32.0147-93.0839)\end{array}$ & $\begin{array}{c}594.2606 \\
(211.1064-1672.8327)\end{array}$ & $\mathrm{NA}^{* * *}$ \\
\hline Cantharidin & $\begin{array}{c}21.9320 \\
(13.3453-36.0436)\end{array}$ & $\begin{array}{c}147.4225 \\
(46.8714-463.6810)\end{array}$ & $\begin{array}{c}2.2175 \\
(0.6116-12.0751)\end{array}$ & $\begin{array}{c}155.0286 \\
(76.7546-313.1261)\end{array}$ & $\begin{array}{c}14.0428 \\
(5.6729-34.7622)\end{array}$ & $\begin{array}{c}14.0622 \\
(8.0013-24.7142)\end{array}$ & $\begin{array}{c}28.5223 \\
(18.6015-43.7340)\end{array}$ & $\begin{array}{c}18.3900 \\
(8.3854-40.3309)\end{array}$ \\
\hline TBZ & $\begin{array}{c}0.5191 \\
(0.0218-12.3413)\end{array}$ & $\begin{array}{c}15.5378 \\
(8.8501-27.2793)\end{array}$ & $\begin{array}{c}3.1998 \\
(1.2133-8.4391)\end{array}$ & $\begin{array}{c}22.3806 \\
(10.4428-47.9653)\end{array}$ & $\begin{array}{c}47.8696 \\
21.2217-107.9790\end{array}$ & $\begin{array}{c}1.2947 \\
(0.6661-2.5165)\end{array}$ & $\begin{array}{c}1268.5889 \\
(110.6555-1453.4930)\end{array}$ & $\begin{array}{c}621.7874 \\
60.1464-6427.9804\end{array}$ \\
\hline
\end{tabular}

* V. m.: Valsa mali, B. b.: Botryosphaeria berengeriana, S. f.: Sclerotinia fructigena, G. c.: Glomerella cingulate, A. a.: Alternaria alternate, S. s.: Sclerotinia sclerotiorum, A. s.: Alternaria solani,

C. s.: Cochliobolus sativum. ${ }^{* *} \mathrm{CI} 95 \%$ : Confidence interval at $95 \%$ probability $(\mu \mathrm{g} / \mathrm{mL}) .{ }^{* * *}$ NA: Not Available. 


\subsubsection{Effect of Various Substituents on the Benzene Ring on Fungistatic Activity}

The presence of different halogen atoms on the benzene ring resulted in various effects on fungistatic activity against the eight fungi tested. As can be seen from Table 2, at the C-3' position of the benzene ring, introduction of the chlorine atom (II-8) produced a more significant increase in the fungistatic activity against all eight fungi tested than introductions of a fluorine atom (II-5) or a bromine atom (II-11). In contrast, the introduction of an iodine atom on the benzene ring (II-14) resulted in a negative influence on fungistatic activity compared with compound II-1 with no substitution on the phenyl ring.

Compounds with substitutions of strongly electron-drawing ( $\mathrm{CN}$, compound II-22; $\mathrm{NO}_{2}$, compounds II-23, 24, 25) or electron-donating $\left(\mathrm{CH}_{3}\right.$, compound II-2; $\mathrm{CH}_{2} \mathrm{CH}_{3}$, compound II-3; $\mathrm{OCH}_{3}$, compounds II-18, 19, 20) groups were found to have a poor antifungal activity. In order to draw firm conclusions about the significance of these data, compounds containing one phenyl ring substituted by two methoxy groups in different positions (compounds II-29, 30) were also tested, and showed the same poor activity. The nitro group was tested in other positions in combination with a methyl group (compound II-31), but only poor spectrum and antifungal activity were detected. Further aromatic groups were also evaluated. Compounds with the phenyl moiety replaced with several kinds of heterocycle produced relatively poor levels of antifungal activity compared to the corresponding compound II-1.

\section{Experiment Section}

\subsection{General Information}

Cantharidin $(\mathrm{CTD}, \geqslant 98 \%$ ) was isolated from $M$. phalerata (Chinese blister beetle), bought from the Chinese herbal medicine market in Xian, China. Norcantharidin (NCTD, $\geqslant 98 \%$ ) was purchased from Alfa Aesar Chemical Co. Ltd., (Haverhill, MA, USA). Dimethyl sulfoxide (DMSO, $\geqslant 99 \%$ ) was obtained from J \& K China Chemical Ltd. (Beijing, China). The fungicide thiabendazole (TBZ, $\geqslant 99.1 \%$ ) was purchased from Sigma-Aldrich Trading Co. Ltd. (Shanghai, China). All reagents and solvents were of reagent grade or purified according to standard methods before use. Analytical thin-layer chromatography (TLC) was performed with silica gel plates using silica gel $60 \mathrm{GF}_{254}$ (Qingdao Haiyang Chemical Co., Ltd., Qingdao, China). Silica gel column chromatography was performed with 200-300 mesh silica gel (Qingdao Haiyang Chemical Co., Ltd.).

Melting points were determined on a WRS-2 apparatus equipped with a microcomputer (Shanghai Precision \& Scientific Instrument Co. Ltd., Shanghai, China) and are uncorrected. Proton nuclear magnetic resonance $\left({ }^{1} \mathrm{H}-\mathrm{NMR}\right)$ spectra and carbon nuclear magnetic resonance $\left({ }^{13} \mathrm{C}-\mathrm{NMR}\right)$ spectra were recorded at 500 and $125 \mathrm{MHz}$, respectively, on a Bruker Avance III $500 \mathrm{MHz} \mathrm{NMR}$ spectrometer (Karlsruhe, Germany) in DMSO- $d_{6}$ using tetramethylsilane (TMS) as the internal standard. High-resolution mass spectrometry (HRMS) was carried out using a Bruker micrOTOF focus II instrument.

\subsection{Synthesis of the Title Compounds II(1-36)}

To a solution of $\operatorname{NCTD~(1,1.0~g,~} 5.95 \mathrm{mmol})$ dissolved in tetrahydrofuran (THF, $10 \mathrm{~mL}$ ) accompanied by triethylamine (TEA, $0.5 \mathrm{~mL}$ ) as the acid-binding agent was added the corresponding aromatic amine (1 equiv., $5.95 \mathrm{mmol}$ ). When the reaction was complete after $14 \mathrm{~h}$ as checked by TLC analysis, the solution was concentrated under reduced pressure and diluted with acetone (100 mL). The resulting filter cake was either recrystallized from methanol or purified by column chromatography $\left(\mathrm{MeOH} / \mathrm{CH}_{2} \mathrm{Cl}_{2}, 1: 4, v / v\right)$ to afford the desired products II (1-36). The yields, physical properties, ${ }^{1} \mathrm{H}-\mathrm{NMR},{ }^{13} \mathrm{C}-\mathrm{NMR}$, and HRMS-ESI of the target compounds II were as follows: 164-165 ${ }^{\circ} \mathrm{C} ;{ }^{1} \mathrm{H}-\mathrm{NMR}$ : 1.47-1.68 (m, 4H, H-1, 2), 2.93-3.12 (m, 2H, H-4, 5), 4.61-4.81 (m, 2H, H-3, 
6) $7.03\left(\mathrm{t}, J=7.41 \mathrm{~Hz}, 1 \mathrm{H}, \mathrm{H}-4^{\prime}\right), 7.29\left(\mathrm{t}, J=7.72 \mathrm{~Hz}, 2 \mathrm{H}, \mathrm{H}-3^{\prime}, 5^{\prime}\right), 7.54\left(\mathrm{~d}, J=7.88 \mathrm{~Hz}, 2 \mathrm{H}, \mathrm{H}-2^{\prime}, 6^{\prime}\right)$,

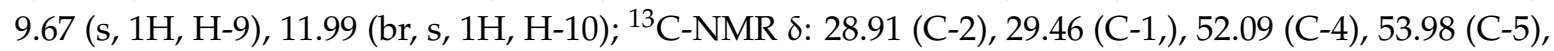
77.33 (C-3), 79.20 (C-6), 123.41 (C-2', C-6' $), 127.28$ (C-4'), 129.06 (C-3', C-5'), $139.75\left(\mathrm{C}-1^{\prime}\right), 169.78$ (C-8), 172.73 (C-7); HR-MS (ESI): $m / z$ calcd. For $\mathrm{C}_{14} \mathrm{H}_{15} \mathrm{NO}_{4} \mathrm{Na}\left([\mathrm{M}+\mathrm{Na}]^{+}\right)$284.0899, found 284.0910.

3-(p-Tolylcarbamoyl)-7-oxabicyclo[2.2.1]heptane-2-carboxylic acid (II-2). Yield 58\%, white solid, mp: 211-212 ${ }^{\circ} \mathrm{C} ;{ }^{1} \mathrm{H}-\mathrm{NMR} \delta: 1.44-1.70(\mathrm{~m}, 4 \mathrm{H}, \mathrm{H}-1,2), 2.25$ (s, 3H, H-7'), $2.94(\mathrm{~d}, J=9.77 \mathrm{~Hz}, 1 \mathrm{H}, \mathrm{H}-4), 3.05$ $(\mathrm{d}, J=9.46 \mathrm{~Hz}, 1 \mathrm{H}, \mathrm{H}-5), 4.64(\mathrm{~d}, J=4.41 \mathrm{~Hz}, 1 \mathrm{H}, \mathrm{H}-6), 4.79(\mathrm{~d}, J=3.78 \mathrm{~Hz}, 1 \mathrm{H}, \mathrm{H}-3), 7.09(\mathrm{~d}, J=8.20 \mathrm{~Hz}$, 2H, H-3', 5'), 7.42 (d, J = $\left.8.20 \mathrm{~Hz}, 2 \mathrm{H}, \mathrm{H}-2^{\prime}, 6^{\prime}\right) 9.56$ (s, 1H, H-9) 11.95 (br, s, 1H, H-10); ${ }^{13} \mathrm{C}-\mathrm{NMR} \delta$ : 20.90 (s, 1C, C-7'), 28.90 (s, 1C, C-1), 29.46 (s, 1C, C-2), 52.07 (s, 1C, C-4), 53.97 (s, 1C, C-5), 77.31 (s, 1C, C-6), 79.22 (s, 1C, C-3), 119.68 (s, 2C, C-2' , C-6'), 129.41 (s, 2C, C-3', C5'), 132.28 (s, 1C, C-1'), 137.24 (s, 1C, C-4'), 169.54 (s, 1C, C-8), 172.72 (s, 1C, C-7); HR-MS (ESI): $m / z$ calcd for $\mathrm{C}_{15} \mathrm{H}_{17} \mathrm{NO}_{4} \mathrm{Na}$ $\left([\mathrm{M}+\mathrm{Na}]^{+}\right)$298.1055, found 298.1051.

3-((2-Ethylphenyl)carbamoyl)-7-oxabicyclo[2.2.1]heptane-2-carboxylic acid (II-3). Yield 80\%, white solid, mp: $172-173{ }^{\circ} \mathrm{C}^{1}{ }^{1} \mathrm{H}-\mathrm{NMR} \delta: 1.15\left(\mathrm{t}, J=7.41 \mathrm{~Hz}, 3 \mathrm{H}, \mathrm{H}-8^{\prime}\right), 1.54-1.73(\mathrm{~m}, 4 \mathrm{H}, \mathrm{H}-1,2), 2.58(\mathrm{q}, J=7.57 \mathrm{~Hz}$, 2H, H-7'), 3.06-3.15 (m, 2H, H-4, 5), $4.74(\mathrm{~d}, J=5.04 \mathrm{~Hz}, 1 \mathrm{H}, \mathrm{H}-6), 4.87(\mathrm{~d}, J=3.47 \mathrm{~Hz}, 1 \mathrm{H}, \mathrm{H}-3)$, 7.05-7.10 (m, 1H, H-4'), 7.13-7.22 (m, 2H, H-3', 5'), 7.66 (d, J = 7.88 Hz, 1H, H-6'), 8.76 (s, 1H, H-9), 12.21 (br, s, 1H, H-10); ${ }^{13} \mathrm{C}-\mathrm{NMR}$ 8: $14.61\left(\mathrm{C}-8^{\prime}\right), 24.04\left(\mathrm{C}-7^{\prime}\right), 28.94$ (C-1, C-2), 52.26 (C-4), 54.60 (C-5), 77.56 (C-3), 79.58 (C-6), $123.85\left(\mathrm{C}-6^{\prime}\right), 124.98\left(\mathrm{C}-3^{\prime}\right), 126.37\left(\mathrm{C}-5^{\prime}\right), 128.84\left(\mathrm{C}-4^{\prime}\right), 135.71\left(\mathrm{C}-2^{\prime}\right)$, 136.30 (C-1'), 169.93 (C-8), 172.82 (C-7); HR-MS (ESI): $m / z$ calcd for $\mathrm{C}_{16} \mathrm{H}_{18} \mathrm{NO}_{4}\left([\mathrm{M}-1]^{-}\right)$288.1236, found 288.1246 .

3-((2-Fluorophenyl)carbamoyl)-7-oxabicyclo[2.2.1]heptane-2-carboxylic acid (II-4). Yield 85\%, white solid, mp: $196-197{ }^{\circ} \mathrm{C} ;{ }^{1} \mathrm{H}-\mathrm{NMR} \delta: 1.53-1.68(\mathrm{~m}, 4 \mathrm{H}, \mathrm{H}-1,2), 3.04(\mathrm{~d}, J=9.46 \mathrm{~Hz}, 1 \mathrm{H}, \mathrm{H}-4), 3.21(\mathrm{~d}, J=9.77 \mathrm{~Hz}$, 1H, H-5), 4.61-4.77 (m, 1H, H-6), 4.78-4.93 (m, 1H, H-3), 7.06-7.19 (m, 2H, H-3' , 5'), 7.20-7.28 (m, 1H, H-4'), 8.02 (t, J = 7.72 Hz, 1H, H-6'), 9.28 (s, 1H, H-9), 12.13 (br, s, 1H, H-10); ${ }^{13} \mathrm{C}-\mathrm{NMR}$ 8: 28.92 (s, 1C, C-1), 29.11 (s, 1C, C-2), 52.28 (s, 1C, C-4), 54.13 (s, 1C, C-5), 77.54 (s, 1C, C-3), 79.36 (s, 1C, C-6), 115.53 (s, 1C, C-3'), $123.24\left(\mathrm{~s}, 1 \mathrm{C}, \mathrm{C}-1^{\prime}\right), 124.83\left(\mathrm{~s}, 1 \mathrm{C}, 6^{\prime}\right), 126.95$ (s, 1C, 4'), 152.24 (s, 1C, C-5'), 154.17 (s, 1C, C-2'), 170.22 (s, 1C, C-8), 172.71 (s, 1C, C-7); HR-MS (ESI): $m / z$ calcd. For $\mathrm{C}_{14} \mathrm{H}_{14} \mathrm{FNO}_{4} \mathrm{Na}\left([\mathrm{M}+\mathrm{Na}]^{+}\right)$ 302.0805, found 302.0807 .

3-((3-Fluorophenyl)carbamoyl)-7-oxabicyclo[2.2.1]heptane-2-carboxylic acid (II-5). Yield 86\%, white solid, mp: $165-167^{\circ} \mathrm{C} ;{ }^{1} \mathrm{H}-\mathrm{NMR} \delta: 1.41-1.71(\mathrm{~m}, 4 \mathrm{H}, \mathrm{H}-1,2), 2.97$ (d, $\left.J=9.46 \mathrm{~Hz}, 1 \mathrm{H}, \mathrm{H}-4\right), 3.07(\mathrm{~d}, J=9.46 \mathrm{~Hz}$, $1 \mathrm{H}, \mathrm{H}-5), 4.66(\mathrm{~d}, J=4.41 \mathrm{~Hz}, 1 \mathrm{H}, \mathrm{H}-6), 4.80(\mathrm{~d}, J=3.78 \mathrm{~Hz}, 1 \mathrm{H}, \mathrm{H}-3), 6.85(\mathrm{td}, J=8.43,2.36 \mathrm{~Hz}, 1 \mathrm{H}$, H-4') $7.22\left(\mathrm{~d}, J=8.20 \mathrm{~Hz}, 1 \mathrm{H}, \mathrm{H}-6^{\prime}\right), 7.29-7.36\left(\mathrm{~m}, 1 \mathrm{H}, \mathrm{H}-5^{\prime}\right), 7.57\left(\mathrm{~d}, J=11.66 \mathrm{~Hz}, 1 \mathrm{H}, \mathrm{H}-2^{\prime}\right), 9.95$ (s,

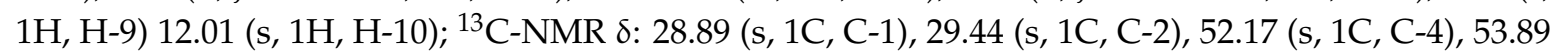
(s, 1C, C-5), 77.38 (s, 1C, C-3), 79.07 (s, 1C, C-6), 106.28 (s, 1C, C-4'), 109.72 (s, 1C, C-2'), 115.31 (s, 1C, C-6'), 130.62 (s, 1C, C-5'), 141.46 (s, 1C, C-1'), 161.65 (s, 1C, C-3'), 170.19 (s, 1C, C-8), 172.64 (s, 1C, C-7); HR-MS (ESI): $m / z$ calcd. For $\mathrm{C}_{14} \mathrm{H}_{14} \mathrm{FNO}_{4} \mathrm{Na}\left([\mathrm{M}+\mathrm{Na}]^{+}\right.$) 302.0805, found 302.0811 .

3-((4-Fluorophenyl)carbamoyl)-7-oxabicyclo[2.2.1]heptane-2-carboxylic acid (II-6). Yield 78\%, white solid, mp: $186-187^{\circ} \mathrm{C} ;{ }^{1} \mathrm{H}-\mathrm{NMR} \delta:$ 1.45-1.69 (m, 4H, H-1, 2), 2.93-2.98 (m, 1H, H-4), 3.02-3.08 (m, 1H, H-5), $4.65(\mathrm{~d}, J=4.10 \mathrm{~Hz}, 1 \mathrm{H}, \mathrm{H}-6), 4.79(\mathrm{~d}, J=3.78 \mathrm{~Hz}, 1 \mathrm{H}, \mathrm{H}-3), 7.13\left(\mathrm{t}, J=8.83 \mathrm{~Hz}, 2 \mathrm{H}, \mathrm{H}-2^{\prime}, 6^{\prime}\right), 7.55(\mathrm{dd}$, $\left.J=8.83,5.04 \mathrm{~Hz}, 2 \mathrm{H}, \mathrm{H}-3^{\prime}, 5^{\prime}\right), 9.73$ (s, 1H, H-8), 11.98 (br, s, 1H, H-7); ${ }^{13} \mathrm{C}-\mathrm{NMR} \delta: 28.90$ (s, 1C, C-1), 29.46 (s, 1C, C-2), 52.15 (s, 1C, C-4), 53.83 (s, 1C, C-5), 77.34 (s, 1C, C-3), 79.10 (s, 2C, C-6), 115.49 (s, 2C, C-3', C-5'), 121.37 (s, 2C, C-2', C-6' $), 136.13\left(\mathrm{~s}, 1 \mathrm{C}, \mathrm{C}-1^{\prime}\right), 157.37$ (s, 1C, C-4'), 169.71 (s, 1C, C-8), 172.68 (s, 1C, C-7); HR-MS (ESI): $m / z$ calcd. For $\mathrm{C}_{14} \mathrm{H}_{14} \mathrm{FNO}_{4} \mathrm{Na}\left([\mathrm{M}+\mathrm{Na}]^{+}\right)$302.0805, found 302.0813 .

3-((2-Chlorophenyl)carbamoyl)-7-oxabicyclo[2.2.1]heptane-2-carboxylic acid (II-7). Yield 71\%, white solid, mp: $177-178{ }^{\circ} \mathrm{C} ;{ }^{1} \mathrm{H}-\mathrm{NMR} \delta: 1.54-1.75(\mathrm{~m}, 4 \mathrm{H}, \mathrm{H}-1,2), 3.15(\mathrm{q}, J=9.77 \mathrm{~Hz}, 2 \mathrm{H}, \mathrm{H}-4,5), 4.78$ (d, $J=4.73 \mathrm{~Hz}, 1 \mathrm{H}, \mathrm{H}-6), 4.91(\mathrm{~d}, J=3.47 \mathrm{~Hz}, 1 \mathrm{H}, \mathrm{H}-3), 7.12\left(\mathrm{t}, J=7.72 \mathrm{~Hz}, 1 \mathrm{H}, \mathrm{H}-4^{\prime}\right), 7.32(\mathrm{t}, J=7.72 \mathrm{~Hz}$, $\left.1 \mathrm{H}, \mathrm{H}-5^{\prime}\right), 7.48\left(\mathrm{~d}, J=7.88 \mathrm{~Hz}, 1 \mathrm{H}, \mathrm{H}-3^{\prime}\right), 8.05\left(\mathrm{~d}, J=7.88 \mathrm{~Hz}, 1 \mathrm{H}, \mathrm{H}-6^{\prime}\right), 9.07$ (s, $\left.1 \mathrm{H}, \mathrm{H}-9\right), 12.30$ (br, s,

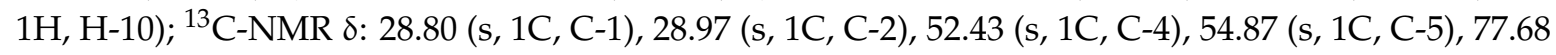


(s, 1C, C-3), 79.49 (s, 1C, C-6), 123.34 (s, 1C, C-6'), 123.95 (s, 1C, C-5'), 125.36 (s, 1C, C-2' ), 127.93 (s, 1C, C-3'), 129.64 (s, 1C, C-4'), 135.54 (s, 1C, C-1'), 170.25 (s, 1C, C-8), 172.61 (s, 1C, C-7); HR-MS (ESI): m/z calcd. For $\mathrm{C}_{14} \mathrm{H}_{14} \mathrm{ClNO}_{4} \mathrm{Na}\left([\mathrm{M}+\mathrm{Na}]^{+}\right)$318.0509, found 318.0513.

3-((3-Chlorophenyl)carbamoyl)-7-oxabicyclo[2.2.1]heptane-2-carboxylic acid (II-8). Yield 55\%, white solid, mp: $178-179{ }^{\circ} \mathrm{C} ;{ }^{1} \mathrm{H}-\mathrm{NMR} \delta: 1.44-1.70(\mathrm{~m}, 4 \mathrm{H}, \mathrm{H}-1,2), 2.93-3.01$ (m, $\left.1 \mathrm{H}, \mathrm{H}-4\right), 3.03-3.13$ (m, $\left.1 \mathrm{H}, \mathrm{H}-5\right)$, $4.66(\mathrm{~d}, J=4.41 \mathrm{~Hz}, 1 \mathrm{H}, \mathrm{H}-6), 4.79(\mathrm{~d}, J=3.78 \mathrm{~Hz}, 1 \mathrm{H}, \mathrm{H}-3), 6.99-7.15\left(\mathrm{~m}, 1 \mathrm{H}, \mathrm{H}-4^{\prime}\right), 7.23-7.43(\mathrm{~m}$, 2H, H-5', 6'), 7.81 (s, 1 H, H-2'), 9.93 (s, 1H, H-9), 12.01 (s, 1H, H-10); ${ }^{13} \mathrm{C}-\mathrm{NMR}$ 8: 28.89 (s, 1C, C-1), 29.44 (s, 1C, C-2), 52.20 (s, 1C, C-4), 53.84 (s, 1C, C-5), 77.38 (s, 1C, C-3), 79.03 (s, 1C, C-6), 117.97 (s, 1C, C-6'), 119.17 (s, 1C, C-2'), 123.09 (s, 1C, C-4'), 130.75 (s, 1C, C-5'), 133.44 (s, 1C, C-3'), 141.18 (s, 1C, C-1'), 170.21 (s, 1C, C-8), 172.63 (s, 1C, C-7); HR-MS (ESI): $m / z$ calcd. For $\mathrm{C}_{14} \mathrm{H}_{14} \mathrm{ClNO}_{4} \mathrm{Na}$ $\left([\mathrm{M}+\mathrm{Na}]^{+}\right)$318.0509, found 318.0515.

3-((4-Chlorophenyl)carbamoyl)-7-oxabicyclo[2.2.1]heptane-2-carboxylic acid (II-9). Yield 56\%, white solid, mp: $178-181^{\circ} \mathrm{C} ;{ }^{1} \mathrm{H}-\mathrm{NMR} \delta: 1.44-1.69(\mathrm{~m}, 4 \mathrm{H}, \mathrm{H}-1,2), 2.97(\mathrm{~d}, J=9.46 \mathrm{~Hz}, 1 \mathrm{H}, \mathrm{H}-4), 3.06(\mathrm{~d}, J=9.46 \mathrm{~Hz}$, $1 \mathrm{H}, \mathrm{H}-5), 4.66(\mathrm{~d}, J=4.41 \mathrm{~Hz}, 1 \mathrm{H}, \mathrm{H}-6), 4.79(\mathrm{~d}, J=3.47 \mathrm{~Hz}, 1 \mathrm{H}, \mathrm{H}-3), 7.35\left(\mathrm{~d}, J=8.51 \mathrm{~Hz}, 2 \mathrm{H}, \mathrm{H}-3^{\prime}, 5^{\prime}\right)$,

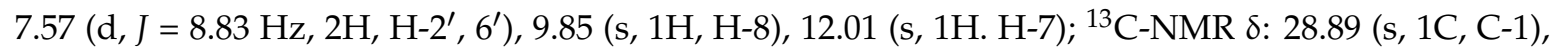
29.45 (s, 1C, C-2), 52.16 (s, 1C, C-4), 53.87 (s, 1C, C-5), 77.36 (s, 1C, C-3), 79.08 (s, 1C, C-6), 121.16 (s, 2C, C-2' $\left.{ }^{\prime} \mathrm{C}-6^{\prime}\right), 126.93$ (s, 1C, C-4'), 128.96 (s, 2C, C-3', $\left.\mathrm{C}-5^{\prime}\right), 138.73$ (s, 1C, C-1'), 169.96 (s, 1C, C-8), 172.66 (s, 1C, C-7); HR-MS (ESI): $m / z$ calcd. For $\mathrm{C}_{14} \mathrm{H}_{14} \mathrm{ClNO}_{4} \mathrm{Na}\left([\mathrm{M}+\mathrm{Na}]^{+}\right)$318.0509, found 318.0519.

3-((2-Bromophenyl)carbamoyl)-7-oxabicyclo[2.2.1]heptane-2-carboxylic acid (II-10). Yield 45\%, white solid, mp: $169-171{ }^{\circ} \mathrm{C} ;{ }^{1} \mathrm{H}-\mathrm{NMR}$ 8: $1.55-1.73$ (m, 4H, H-1, 2), 3.14 (s, 2H, H-4, 5), 4.79 (d, J = $4.73 \mathrm{~Hz}$, $1 \mathrm{H}, \mathrm{H}-6), 4.91(\mathrm{~d}, J=2.84 \mathrm{~Hz}, 1 \mathrm{H}, \mathrm{H}-3), 7.06\left(\mathrm{t}, J=7.57 \mathrm{~Hz}, 1 \mathrm{H}, \mathrm{H}-4^{\prime}\right), 7.30-7.40\left(\mathrm{~m}, 1 \mathrm{H}, \mathrm{H}-5^{\prime}\right), 7.63(\mathrm{~d}$, $\left.J=7.88 \mathrm{~Hz}, 1 \mathrm{H}, \mathrm{H}-3^{\prime}\right), 7.99\left(\mathrm{~d}, J=7.88 \mathrm{~Hz}, 1 \mathrm{H}, \mathrm{H}-6^{\prime}\right), 8.99$ (s, 1H, H-9' $), 12.29$ (br, s, 1H, H-10); ${ }^{13} \mathrm{C}-\mathrm{NMR}$ 8: 28.80 (s, 1C, C-1), 28.99 (s, 1C, C-2), 52.41 (s, 1C, C-4), 54.89 (s, 1C, C-5), 77.67 (s, 1C, C-3), 79.45 (s, 1C, C-6), 114.90 (s, 1C, C-2'), 123.93 (s, 1C, C-6'), 125.99 (s, 1C, C-5'), 128.46 (s, 1C, C-3'), 132.89 (s, 1C, C-4'), 136.77 (s, 1C, C-1' $), 170.24$ (s, 1C, C-8), 172.58 (s, 1C, C-7); HR-MS (ESI): $m / z$ calcd. For $\mathrm{C}_{14} \mathrm{H}_{14} \mathrm{BrNO}_{4} \mathrm{Na}\left([\mathrm{M}+\mathrm{Na}]^{+}\right)$362.0004, found 362.0008.

3-((3-Bromophenyl)carbamoyl)-7-oxabicyclo[2.2.1]heptane-2-carboxylic acid (II-11). Yield 56\%, white solid, mp: 189-191 ${ }^{\circ} \mathrm{C} ;{ }^{1} \mathrm{H}-\mathrm{NMR}$ $8: 1.45-1.70$ (m, 4H, H-1, 2), 2.94-3.01 (m, 1H, H-4), 3.03-3.09 (m, 1H, H-5), 4.67 (br, s, 1H, H-6), 4.79 (br, s, 1H, H-3), 7.19-7.30 (m, 2H, H-5', 6'), 7.39 (d, J = 7.88 Hz, 1H, H-4'), 7.96 (br, s, 1H, H-2'), 9.91 (br, s, 1H, H-9), 12.01 (s, 1H, H-10); ${ }^{13} \mathrm{C}-\mathrm{NMR}$ 8: 28.90 (s, 1C, C-1), 29.44 (s, 1C, C-2), 52.21 (s, 1C, C-4), 53.84 (s, 1C, C-5), 77.40 (s, 1C, C-3), 79.03 (s, 1C, C-6), 118.36 (s, 1C, C-3'), $121.94\left(\mathrm{~s}, 1 \mathrm{C}, \mathrm{C}-6^{\prime}\right), 122.05$ (s, 1C, C-2' $), 125.99$ (s, 1C, C-4' $), 131.06\left(\mathrm{~s}, 1 \mathrm{C}, \mathrm{C}-5^{\prime}\right), 141.32\left(\mathrm{~s}, 1 \mathrm{C}, \mathrm{C}-1^{\prime}\right)$, 170.19 (s, 1C, C-8), 172.63 (s, 1C, C-7); HR-MS (ESI): $m / z$ calcd. For $\mathrm{C}_{14} \mathrm{H}_{14} \mathrm{BrNO}_{4} \mathrm{Na}\left([\mathrm{M}+\mathrm{Na}]^{+}\right)$ 362.0004, found 362.0011 .

3-((4-Bromophenyl)carbamoyl)-7-oxabicyclo[2.2.1]heptane-2-carboxylic acid (II-12). Yield 65\%, white solid, mp: $188-192{ }^{\circ} \mathrm{C} ;{ }^{1} \mathrm{H}-\mathrm{NMR} \delta: 1.44-1.69(\mathrm{~m}, 4 \mathrm{H}, \mathrm{H}-1,2), 2.93-3.00(\mathrm{~m}, 1 \mathrm{H}, \mathrm{H}-4), 3.06(\mathrm{~d}, J=9.46 \mathrm{~Hz}, 1 \mathrm{H}$, H-5), 4.66 (d, J = 4.41 Hz, 1H, H-6), 4.79 (d, J = 3.78 Hz, 1H, H-3), 7.44-7.49 (m, 2H, H-3', 5' ), 7.50-7.56 (m, 2H, H-2' , 6'), 9.85 (s, 1H, H-9), 12.01 (s, 1H, H-10); ${ }^{13} \mathrm{C}-\mathrm{NMR}$ 8: 28.89 (s, 1C, C-1), 29.45 (s, 1C, C-2), 52.16 (s, 1C, C-4), 53.88 (s, 1C, C-5), 77.36 (s, 1C, C-3), 79.07 (s, 1C, C-6), 114.90 (s, 1C, C-4'), 121.55 (s, 2C, C-2', C-6'), 131.87 (s, 2C, C-3', C-5'), 139.14 (s, 1C, C-1'), 169.98 (s, 1C, C-8), 172.66 (s, 1C, C-7); HR-MS (ESI): $m / z$ calcd. For $\mathrm{C}_{14} \mathrm{H}_{14} \mathrm{BrNO}_{4} \mathrm{Na}\left([\mathrm{M}+\mathrm{Na}]^{+}\right)$362.0004, found 362.0015.

3-((2-Iodophenyl)carbamoyl)-7-oxabicyclo[2.2.1]heptane-2-carboxylic acid (II-13). Yield 74\%, white solid, mp: 178-180 ${ }^{\circ} \mathrm{C} ;{ }^{1} \mathrm{H}-\mathrm{NMR}$ $:$ : 1.54-1.75 (m, 4H, H-1, 2), 3.12 (br, s, 2H, H-3, 6), 4.81 (br, s, 1H, H-6), 4.91 (br, s, 1H, H-3), $6.91\left(\mathrm{t}, J=7.09 \mathrm{~Hz}, 1 \mathrm{H}, \mathrm{H}-4^{\prime}\right), 7.37\left(\mathrm{t}, J=7.25 \mathrm{~Hz}, 1 \mathrm{H}, \mathrm{H}-5^{\prime}\right), 7.77(\mathrm{~d}, J=7.57 \mathrm{~Hz}, 1 \mathrm{H}$,

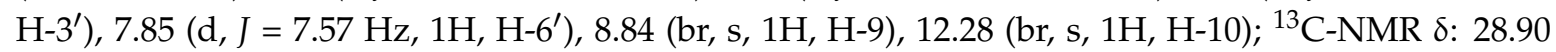
(1C, C-1), 29.02 (1C, C-2), 52.35 (1C, C-4), 54.66 (1C, C-5), 77.61 (1C, C-3), 79.33 (1C, C-6), 124.56 (1C, C-2'), $126.81\left(1 \mathrm{C}, \mathrm{C}-6^{\prime}\right), 128.98\left(1 \mathrm{C}, \mathrm{C}-4^{\prime}\right), 139.32\left(1 \mathrm{C}, \mathrm{C}-5^{\prime}\right), 139.74\left(1 \mathrm{C}, \mathrm{C}-3^{\prime}\right), 170.16(1 \mathrm{C}, \mathrm{C}-8), 172.58$ (1C, C-7); HR-MS (ESI): $m / z$ calcd. For $\mathrm{C}_{14} \mathrm{H}_{14} \mathrm{INO}_{4} \mathrm{Na}\left([\mathrm{M}+\mathrm{Na}]^{+}\right)$409.9865, found 409.9863 . 
3-((3-Iodophenyl)carbamoyl)-7-oxabicyclo[2.2.1]heptane-2-carboxylic acid (II-14). Yield 54\%, white solid, mp: $174-175^{\circ} \mathrm{C} ;{ }^{1} \mathrm{H}-\mathrm{NMR} \delta: 1.41-1.71(\mathrm{~m}, 4 \mathrm{H}, \mathrm{H}-1,2), 2.97(\mathrm{~d}, J=9.46 \mathrm{~Hz}, 1 \mathrm{H}, \mathrm{H}-4), 3.07(\mathrm{~d}, J=9.46 \mathrm{~Hz}$, $1 \mathrm{H}, \mathrm{H}-5), 4.66(\mathrm{~d}, J=4.41 \mathrm{~Hz}, 1 \mathrm{H}, \mathrm{H}-6), 4.80(\mathrm{~d}, J=3.78 \mathrm{~Hz}, 1 \mathrm{H}, \mathrm{H}-3), 6.85(\mathrm{td}, J=8.43,2.36 \mathrm{~Hz}, 1 \mathrm{H}$, $\left.\mathrm{H}-4^{\prime}\right), 7.22\left(\mathrm{~d}, J=8.20 \mathrm{~Hz}, 1 \mathrm{H}, \mathrm{H}-6^{\prime}\right), 7.29-7.36\left(\mathrm{~m}, 1 \mathrm{H}, \mathrm{H}-5^{\prime}\right), 7.57\left(\mathrm{~d}, J=11.66 \mathrm{~Hz}, 1 \mathrm{H}, \mathrm{H}-2^{\prime}\right), 9.95$ (s, 1H, H-9) 12.01 (s, 1H, H-10); ${ }^{13} \mathrm{C}-\mathrm{NMR} \delta: 28.89$ (s, 1C, C-1), 29.44 (s, 1C, C-2), 52.17 (s, 1C, C-4), 53.89 (s, 1C, C-5), 77.38 (s, 1C, C-3), 79.07 (s, 1C, C-6), 106.28 (s, 1C, C-4'), 109.72 (s, 1C, C-2'), 115.31 (s, 1C, C-6'), 130.62 (s, 1C, C-5'), 141.46 (s, 1C, C-1'), 161.65 (s, 1C, C-3'), 170.19 (s, 1C, C-8), 172.64 (s, 1C, C-7); HR-MS (ESI): $m / z$ calcd. For $\mathrm{C}_{14} \mathrm{H}_{14} \mathrm{INO}_{4} \mathrm{Na}\left([\mathrm{M}+\mathrm{Na}]^{+}\right)$409.9865, 409.9871.

3-((3-Iodophenyl)carbamoyl)-7-oxabicyclo[2.2.1]heptane-2-carboxylic acid (II-15). Yield 81\%, white solid, mp: $171-172{ }^{\circ} \mathrm{C} ;{ }^{1} \mathrm{H}-\mathrm{NMR} \delta: 1.47-1.69(\mathrm{~m}, 4 \mathrm{H}, \mathrm{H}-1,2), 2.96(\mathrm{~d}, J=9.46 \mathrm{~Hz}, 1 \mathrm{H}, \mathrm{H}-4), 3.05(\mathrm{~d}, J=9.46 \mathrm{~Hz}$, 1H, H-5), 4.65 (br, s, 1H, H-6), 4.79 (br, s, 1H, H-3), 7.39 (d, J = $\left.8.51 \mathrm{~Hz}, 2 \mathrm{H}, \mathrm{H}-3^{\prime}, 5^{\prime}\right), 7.62$ (d, J = $8.51 \mathrm{~Hz}$, 2H, H-2' $6^{\prime}$ ), 9.81 (br, s, 1H, H-9), 12.01 (br, s, 1H, H-10); ${ }^{13} \mathrm{C}-\mathrm{NMR}$ 8: 28.90 (1C, C-1), 29.44 (1C, C-2), 52.19 (1C, C-4), 53.93 (1C, C-5), 77.38 (1C, C-3), 79.06 (1C, C-6), 86.66 (1C, C-4'), 121.86 (2C, C-2' , C-6'), 137.69 (2C, C-3', C-5'), 139.61 (1C, C-1'), 169.98 (1C, C-8), 172.65 (1C, C-7); HR-MS (ESI): $m / z$ calcd. For $\mathrm{C}_{14} \mathrm{H}_{14} \mathrm{INO}_{4} \mathrm{Na}\left([\mathrm{M}+\mathrm{Na}]^{+}\right)$409.9865, found 409.9871 .

3-((4-Hydroxyphenyl)carbamoyl)-7-oxabicyclo[2.2.1]heptane-2-carboxylic acid (II-16). Yield 56\%, white solid, mp: $174-175^{\circ} \mathrm{C} ;{ }^{1} \mathrm{H}-\mathrm{NMR} \delta: 1.42-1.74(\mathrm{~m}, 4 \mathrm{H}, \mathrm{H}-1,2) 2.89-2.96$ (m, $\left.1 \mathrm{H}, \mathrm{H}-4\right) 3.02$ (d, J = $9.46 \mathrm{~Hz}$, $1 \mathrm{H}, \mathrm{H}-5) 4.62(\mathrm{~d}, J=3.78 \mathrm{~Hz}, 1 \mathrm{H}, \mathrm{H}-6) 4.79(\mathrm{~d}, J=2.52 \mathrm{~Hz}, 1 \mathrm{H}, \mathrm{H}-3) 6.68\left(\mathrm{~d}, J=8.20 \mathrm{~Hz}, 2 \mathrm{H}, \mathrm{H}-3^{\prime}\right.$, 5') 7.30 (d, J = $\left.8.51 \mathrm{~Hz}, 2 \mathrm{H}, \mathrm{H}-2^{\prime}, 6^{\prime}\right) 9.13$ (br, s, 1H, H-7') 9.36 (s, 1H, H-9) 11.89 (br, s, 1H, H-10); ${ }^{13} \mathrm{C}-\mathrm{NMR}$ 8: 28.89 (s, 1C, C-1), 29.47 (s, 1C, C-2), 52.03 (s, 1C, C-4), 53.89 (s, 1C, C-5), 77.26 (s, 1C, C-3), 79.24 (s, 1C, C-6), 115.39 (s, 2C, C-3' , C-5'), 121.49 (s, 2C, C-2' , C-6' $), 131.39$ (s, 1C, C-1'), 153.62 (s, 1C, C-4'), 169.13 (s, 1C, C-8), 172.77 (s, 1C, C-7); HR-MS (ESI): $m / z$ calcd for $\mathrm{C}_{14} \mathrm{H}_{15} \mathrm{NO}_{5} \mathrm{Na}\left([\mathrm{M}+\mathrm{Na}]^{+}\right)$ 300.0848, found 300.0851.

3-((4-Carboxyphenyl)carbamoyl)-7-oxabicyclo[2.2.1]heptane-2-carboxylic acid (II-17). Yield 44\%, white solid, mp: $256-257{ }^{\circ} \mathrm{C} ;{ }^{1} \mathrm{H}-\mathrm{NMR}$ 8: 1.52-1.64 (m, 4H, H-1, 2), $2.98(\mathrm{~d}, J=9.46 \mathrm{~Hz}, 1 \mathrm{H}, \mathrm{H}-4), 3.11(\mathrm{~d}$, $J=9.77 \mathrm{~Hz}, 1 \mathrm{H}, \mathrm{H}-5), 4.68(\mathrm{~d}, J=4.41 \mathrm{~Hz}, 1 \mathrm{H}, \mathrm{H}-6), 4.80(\mathrm{~d}, J=3.78 \mathrm{~Hz}, 1 \mathrm{H}, \mathrm{H}-3), 7.66(\mathrm{~d}, J=8.83 \mathrm{~Hz}$, 2H, H-2', $\left.6^{\prime}\right), 7.89$ (d, J = $\left.8.83 \mathrm{~Hz}, 2 \mathrm{H}, \mathrm{H}-3^{\prime}, 5^{\prime}\right), 10.05$ (s, 1H, H-9), 12.35 (br, s, 2H, H-10); ${ }^{13} \mathrm{C}-\mathrm{NMR}$ 8: 28.91 (s, 1C, C-1) 29.44 (s, 1C, C-2) 52.20 (s, 1C, C-4) 53.97 (s, 1C, C-5) 77.42 (s, 1C, C-3) 79.11 (s, 1C, C-6) 118.80 (s, 2C, C-2', C-6') 125.29 (s, 1C, C-4') 130.78 (s, 2C, C-3', C-5') 143.69 (m, 1C, C-1') $167.32\left(\mathrm{~m}, 1 \mathrm{C}, \mathrm{C}-7^{\prime}\right) 170.32$ (s, 1C, C-8) 172.65 (s, 1C, C-7); HR-MS (ESI): $m / z$ calcd for $\mathrm{C}_{15} \mathrm{H}_{15} \mathrm{NO}_{6} \mathrm{Na}$ $\left([\mathrm{M}+\mathrm{Na}]^{+}\right)$328.0797, found 328.0796.

3-((2-Methoxyphenyl)carbamoyl)-7-oxabicyclo[2.2.1]heptane-2-carboxylic acid (II-18). Yield 66\%white solid, mp: 151-152 ${ }^{\circ} \mathrm{C} ;{ }^{1} \mathrm{H}-\mathrm{NMR}$ 8 : 1.52-1.72 (m, 4H, H-1, 2), 3.05-3.17 (m, 2H, H-4, 5), 3.83 (s, $\left.3 \mathrm{H}, \mathrm{H}-7^{\prime}\right), 4.71\left(\mathrm{~d}, J=5.04 \mathrm{~Hz}, 1 \mathrm{H}, \mathrm{H}-6^{\prime}\right), 4.89\left(\mathrm{~d}, J=3.47 \mathrm{~Hz}, 1 \mathrm{H}, \mathrm{H}-3^{\prime}\right), 6.86-6.93\left(\mathrm{~m}, 1 \mathrm{H}, \mathrm{H}-5^{\prime}\right)$, $7.02\left(\mathrm{~d}, J=4.10 \mathrm{~Hz}, 2 \mathrm{H}, \mathrm{H}-3^{\prime}, 4^{\prime}\right), 8.11\left(\mathrm{~d}, J=7.88 \mathrm{~Hz}, 1 \mathrm{H}, \mathrm{H}-6^{\prime}\right), 8.87$ (s, 1H, H-9), 12.20 (br, s, 1H, H-10); ${ }^{13}$ C-NMR 8: 28.96 (C-1, C-2), 52.44 (C-7'), 55.32 (C-4), 56.45 (C-5), 77.52 (C-3), 79.54 (C-6), 111.44 (C-3'), $120.32\left(\mathrm{C}-6^{\prime}\right), 120.79\left(\mathrm{C}-5^{\prime}\right), 123.84\left(\mathrm{C}-3^{\prime}\right), 128.31\left(\mathrm{C}-4^{\prime}\right), 148.84\left(\mathrm{C}-2^{\prime}\right), 169.95$ (C-8), 172.61 (C-7); HR-MS (ESI): $m / z$ calcd for $\mathrm{C}_{15} \mathrm{H}_{17} \mathrm{NO}_{5} \mathrm{Na}\left([\mathrm{M}+\mathrm{Na}]^{+}\right) 314.1004$, found 314.1008 .

3-((3-Methoxyphenyl)carbamoyl)-7-oxabicyclo[2.2.1]heptane-2-carboxylic acid (II-19). Yield 73\%, white solid, mp: $165-166{ }^{\circ} \mathrm{C} ;{ }^{1} \mathrm{H}-\mathrm{NMR} \delta: 1.47-1.70(\mathrm{~m}, 4 \mathrm{H}, \mathrm{H}-1,2), 2.95$ (d, J = 9.46 Hz, 1H, H-4), 3.07 $(\mathrm{d}, J=9.77 \mathrm{~Hz}, 1 \mathrm{H}, \mathrm{H}-5), 4.64(\mathrm{~d}, J=4.41 \mathrm{~Hz}, 1 \mathrm{H}, \mathrm{H}-6), 4.80$ (d, J = 3.78 Hz, 1H, H-3), 6.61 (dd, J = 8.20, $\left.1.89 \mathrm{~Hz}, 1 \mathrm{H}, \mathrm{H}-4^{\prime}\right), 7.05\left(\mathrm{~d}, J=8.20 \mathrm{~Hz}, 1 \mathrm{H}, \mathrm{H}-6^{\prime}\right), 7.15-7.22\left(\mathrm{~m}, 1 \mathrm{H}, \mathrm{H}-5^{\prime}\right), 7.29\left(\mathrm{~s}, 1 \mathrm{H}, \mathrm{H}-2^{\prime}\right), 9.68(\mathrm{~s}, 1 \mathrm{H}$,

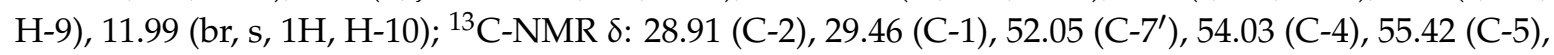
77.34 (C-3), 79.23 (C-6), 105.36 (C-6'), 108.97 (C-2'), 111.89 (C-4'), 129.81 (C-5'), $140.95\left(\mathrm{C}-1^{\prime}\right), 159.95$ (C-3'), 169.83 (C-8), 172.72 (C-7); HR-MS (ESI): $m / z$ calcd for $\mathrm{C}_{15} \mathrm{H}_{17} \mathrm{NO}_{5} \mathrm{Na}\left([\mathrm{M}+\mathrm{Na}]^{+}\right)$314.1004, found 314.0983 .

3-((4-Methoxyphenyl)carbamoyl)-7-oxabicyclo[2.2.1]heptane-2-carboxylic acid (II-20). Yield 71\%, white solid, mp: $167-168{ }^{\circ} \mathrm{C} ;{ }^{1} \mathrm{H}-\mathrm{NMR}: 1.46-1.69$ (m, 4H, H-1, 2), 2.94 (d, J = 9.77 Hz, 1H, H-4), 3.03 (d, 
$J=9.46 \mathrm{~Hz}, 1 \mathrm{H}, \mathrm{H}-5), 4.63(\mathrm{~d}, J=4.10 \mathrm{~Hz}, 1 \mathrm{H}, 1 \mathrm{H}, \mathrm{H}-6), 4.79(\mathrm{~d}, J=3.78 \mathrm{~Hz}, 1 \mathrm{H}, 1 \mathrm{H}, \mathrm{H}-3), 6.87(\mathrm{~d}$, $\left.J=8.83 \mathrm{~Hz}, 2 \mathrm{H}, \mathrm{H}-3^{\prime}, 5^{\prime}\right), 7.44\left(\mathrm{~d}, J=8.83 \mathrm{~Hz}, 2 \mathrm{H}, \mathrm{H}-2^{\prime}, 6^{\prime}\right), 9.51$ (s, 1H, H-9), 11.93 (br, s, 1H, H-10); ${ }^{13}$ C-NMR 8: 28.89 (C-2), 29.47 (C-1), 52.07 (C-7'), 53.87 (C-4), 55.64 (C-5), 77.29 (C-3), 79.20 (C-6), $114.20\left(\mathrm{C}-3^{\prime}, \mathrm{C}^{-} 5^{\prime}\right), 121.24\left(\mathrm{C}-2^{\prime}, \mathrm{C}-6^{\prime}\right), 132.91\left(\mathrm{C}-1^{\prime}\right), 155.54\left(\mathrm{C}-4^{\prime}\right), 169.33$ (C-8), 172.76 (C-7); HR-MS (ESI): $m / z$ calcd for $\mathrm{C}_{15} \mathrm{H}_{17} \mathrm{NO}_{5} \mathrm{Na}\left([\mathrm{M}+\mathrm{Na}]^{+}\right) 314.1004$, found 314.1025 .

3-((4-(Trifluoromethoxy)phenyl)carbamoyl)-7-oxabicyclo[2.2.1]heptane-2-carboxylic acid (II-21). Yield 47\%, yellow solid, mp: $155-156{ }^{\circ} \mathrm{C} ;{ }^{1} \mathrm{H}-\mathrm{NMR}$ : : 1.46-1.69 (m, 4H, H-1, 2), 2.95-3.00 (m, 1H, H-4), 3.07 (d, $J=9.77 \mathrm{~Hz}, 1 \mathrm{H}, \mathrm{H}-5), 4.67(\mathrm{~d}, J=4.41 \mathrm{~Hz}, 1 \mathrm{H}, \mathrm{H}-6), 4.80(\mathrm{~d}, J=3.78 \mathrm{~Hz}, 1 \mathrm{H}, \mathrm{H}-3), 7.30(\mathrm{~d}, J=8.83 \mathrm{~Hz}$, 2H, H-3' $\left.5^{\prime}\right), 7.65$ (d, J = $\left.8.83 \mathrm{~Hz}, 2 \mathrm{H}, \mathrm{H}-2^{\prime}, 6^{\prime}\right), 9.91$ (s, 1H, H-9), 12.01 (s, 1H, H-10); ${ }^{13} \mathrm{C}-\mathrm{NMR} \delta: 28.84$ (m, 1C, C-1), 29.44 (m, 1C, C-2), 52.17 (m, 1C, C-4), 53.89 (m, 1C, C-5), 77.34 (m, 1C, C-3), 79.07 (m, 1C, C-6), 120.88 (s, 2C, C-3', C-5'), 121.65 (s, 1C, C-7'), 121.97 (s, 2C, C-2' , C-6' $), 138.91$ (m, 1C, C-1' $), 143.80$ $\left(\mathrm{m}, 1 \mathrm{C}, \mathrm{C}-4^{\prime}\right), 169.85$ (m, 1C, C-8), 172.47 (m, 1C, C-7); HR-MS (ESI): $\mathrm{m} / z$ calcd for $\mathrm{C}_{15} \mathrm{H}_{14} \mathrm{~F}_{3} \mathrm{NO}_{5} \mathrm{Na}$ $\left([\mathrm{M}+\mathrm{Na}]^{+}\right)$368.0722, found 368.0732 .

3-((4-Cyanophenyl)carbamoyl)-7-oxabicyclo[2.2.1]heptane-2-carboxylic acid (II-22). Yield, 73\%, yellow solid, mp: $164-166{ }^{\circ} \mathrm{C} ;{ }^{1} \mathrm{H}-\mathrm{NMR} \delta: ~ 1.46-1.70(\mathrm{~m}, 4 \mathrm{H}, \mathrm{H}-1,2), 3.00$ (d, J = 9.46 Hz, 1H, H-4), 3.09 $(\mathrm{d}, J=9.77 \mathrm{~Hz}, 1 \mathrm{H}, \mathrm{H}-5), 4.69$ (d, $J=2.52 \mathrm{~Hz}, 1 \mathrm{H}, \mathrm{H}-6), 4.80$ (br, s, 1H, H-3), $7.74\left(\mathrm{~s}, 2 \mathrm{H}, \mathrm{H}-3^{\prime}, 5^{\prime}\right)$, 7.77-7.79 (m, 2H, H-2' , 6' ), 10.20 (s, 1H, H-9), 12.05 (s, 1H, H-10); ${ }^{13} \mathrm{C}-\mathrm{NMR}$ 8: 28.91 (s, 1C, C-1) 29.42 (s, 1C, C-2) 52.33 (s, 1C, C-4) 53.88 (m, 1C, C-5) 77.49 (s, 1C, C-3) 78.97 (s, 1C, C-6) 105.05 (s, 1C, C-4') 119.56 (s, 1C, C-7') 133.65 (s, 2C, C-2', C-6') 143.99 (s, 2C, C-3', C-5') 170.62 (s, 1C, C-8) 172.57 (s, 1C, C-7); HR-MS (ESI): $m / z$ calcd for $\mathrm{C}_{15} \mathrm{H}_{14} \mathrm{~N}_{2} \mathrm{O}_{4} \mathrm{Na}\left([\mathrm{M}+\mathrm{Na}]^{+}\right) 309.0851$,found 309.0856 .

3-((2-Nitrophenyl)carbamoyl)-7-oxabicyclo[2.2.1]heptane-2-carboxylic acid (II-23). Yield, 47\%, yellow solid, mp: $155-156{ }^{\circ} \mathrm{C} ;{ }^{1} \mathrm{H}-\mathrm{NMR}$ 8: $1.46-1.72(\mathrm{~m}, 4 \mathrm{H}, \mathrm{H}-1,2), 2.98-3.12(\mathrm{~m}, 2 \mathrm{H}, \mathrm{H}-4,5), 4.63-4.85$ $(\mathrm{m}, 2 \mathrm{H}, \mathrm{H}-3,6), 7.60\left(\mathrm{t}, J=8.20 \mathrm{~Hz}, 1 \mathrm{H}, \mathrm{H}-4^{\prime}\right), 7.82\left(\mathrm{~d}, J=7.88 \mathrm{~Hz}, 1 \mathrm{H}, \mathrm{H}-5^{\prime}\right), 7.90(\mathrm{~d}, J=7.88 \mathrm{~Hz}$, 1H, H-6'), 8.65 (br, s, 1H, H-3'), 10.28 (br, s, 1H, H-9), 12.02-12.09 (m, 1H, H-10); ${ }^{13} \mathrm{C}-\mathrm{NMR}$ 8: 28.91 (C-2), 29.44 (C-1), 52.24 (C-4), 53.82 (C-5), 77.49 (C-3), 79.00 (C-6), 113.68 (C-6'), 117.96 (C-4'), 125.55 (C-3'), $130.53\left(\mathrm{C}-5^{\prime}\right), 140.91$ (C-1'), 148.42 (C-2'), 170.61 (C-8), 172.63 (C-7); HR-MS (ESI): $m / z$ calcd for $\mathrm{C}_{14} \mathrm{H}_{14} \mathrm{~N}_{2} \mathrm{O}_{6} \mathrm{Na}\left([\mathrm{M}+\mathrm{Na}]^{+}\right)$329.0750, found 329.0754.

3-((3-Nitrophenyl)carbamoyl)-7-oxabicyclo[2.2.1]heptane-2-carboxylic acid (II-24). Yield, 44\%, yellow solid, mp: $178-179{ }^{\circ} \mathrm{C} ;{ }^{1} \mathrm{H}-\mathrm{NMR}$ $8: 1.45-1.70$ (m, 4H, H-1, 2), 2.94-3.01 (m, 1H, H-4), 3.03-3.09 (m, 1H, H-5), 4.67 (br, s, 1H, H-6), 4.79 (br, s, 1H, H-3), 7.19-7.30 (m, 2H, H-4', 6'), 7.39 (d, J = 7.88 Hz, 1H, H-5'), 7.96 (br, s, 1H, H-2'), 9.91 (br, s, 1H, H-9) 12.01 (s, 1H, H-10); ${ }^{13} \mathrm{C}-\mathrm{NMR}$ 8: 28.54 (C-2), 29.36 (C-1), 52.44 (C-4), 53.79 (C-5), 77.80 (C-3), 79.55 (C-6), 115.24 (C-2'), 119.30 (C-4'), $126.72\left(\mathrm{C}-6^{\prime}\right), 130.81$ $\left(\mathrm{C}-5^{\prime}\right), 140.68\left(\mathrm{C}-1^{\prime}\right), 149.11$ (C-3'); HR-MS (ESI): $m / z$ calcd for $\mathrm{C}_{14} \mathrm{H}_{14} \mathrm{~N}_{2} \mathrm{O}_{6} \mathrm{Na}\left([\mathrm{M}+\mathrm{Na}]^{+}\right)$329.0750, found 329.0771 .

3-((4-Nitrophenyl)carbamoyl)-7-oxabicyclo[2.2.1]heptane-2-carboxylic acid (II-25). Yield, 54\%, yellow solid, mp: $156-157{ }^{\circ} \mathrm{C} ;{ }^{1} \mathrm{H}-\mathrm{NMR} \delta: 1.48-1.70(\mathrm{~m}, 4 \mathrm{H}, \mathrm{H}-1,2), 3.02(\mathrm{~d}, J=9.46 \mathrm{~Hz}, 1 \mathrm{H}, \mathrm{H}-4), 3.12$ (d, $J=9.46 \mathrm{~Hz}, 1 \mathrm{H}, \mathrm{H}-5), 4.71$ (br, s, 1H, H-6), 4.81 (br, s, 1H, H-3), 7.80 (d, J = 8.83 Hz, 2H, H-2', $6^{\prime}$ ), $8.22\left(\mathrm{~d}, J=8.83 \mathrm{~Hz}, 2 \mathrm{H}, \mathrm{H}-3^{\prime}, 5^{\prime}\right), 10.40(\mathrm{~s}, 1 \mathrm{H}, \mathrm{H}-9), 12.09$ (br, s, 1H, H-10); ${ }^{13} \mathrm{C}-\mathrm{NMR}$ 8: 28.92 (C-2), 29.42 (C-1), 52.39 (C-4), 53.89 (C-5), 77.56 (C-3), 78.96 (C-6), 119.15 (C-2' , C-6' $), 125.40$ (C-3', C-5'), $142.41\left(\mathrm{C}-4^{\prime}\right), 146.01\left(\mathrm{C}-1^{\prime}\right), 170.79$ (C-8), 172.56 (C-7); HR-MS (ESI): $m / z$ calcd for $\mathrm{C}_{14} \mathrm{H}_{14} \mathrm{~N}_{2} \mathrm{O}_{6} \mathrm{Na}$ $\left([\mathrm{M}+\mathrm{Na}]^{+}\right)$329.0750, found 329.0763.

3-((3,4-Dichlorophenyl)carbamoyl)-7-oxabicyclo[2.2.1]heptane-2-carboxylic acid (II-26). Yield, 88\%, white solid, mp: $160-161{ }^{\circ} \mathrm{C} ;{ }^{1} \mathrm{H}-\mathrm{NMR} \delta: ~ 1.45-1.69$ (m, 4H, H-1, 2), 2.95-3.01 (m, 1H, H-4), 3.02-3.08 (m, 1H, H-5), $4.67(\mathrm{~d}, J=3.78 \mathrm{~Hz}, 1 \mathrm{H}, \mathrm{H}-6), 4.79(\mathrm{~d}, J=2.84 \mathrm{~Hz}, 1 \mathrm{H}, \mathrm{H}-3), 7.40\left(\mathrm{~d}, J=8.83 \mathrm{~Hz}, 1 \mathrm{H}, \mathrm{H}-5^{\prime}\right), 7.55$ $\left(\mathrm{d}, J=8.83 \mathrm{~Hz}, 1 \mathrm{H}, \mathrm{H}-6^{\prime}\right), 7.99$ (s, 1H, H-2'), 10.06 (s, 1H, H-9), 12.04 (s, 1H, H-10); ${ }^{13}$ C-NMR $\delta: 28.86$ $(\mathrm{m}, 1 \mathrm{C}), 29.43(\mathrm{~s}, 1 \mathrm{C}), 52.26(\mathrm{~s}, 1 \mathrm{C}), 53.78(\mathrm{~s}, 1 \mathrm{C}), 77.42(\mathrm{~s}, 1 \mathrm{C}), 78.95(\mathrm{~s}, 1 \mathrm{C}), 119.64(\mathrm{~s}, 1 \mathrm{C}), 120.86(\mathrm{~s}$, 1C), 124.77 (s, 1C), 131.02 (s, 1C), 131.32 (s, 1C), 139.80 (s, 1C), 170.29 (s, 1C), 172.61 (s, 1C); HR-MS (ESI): $m / z$ calcd for $\mathrm{C}_{14} \mathrm{H}_{13} \mathrm{C}_{12} \mathrm{NO}_{4} \mathrm{Na}\left([\mathrm{M}+\mathrm{Na}]^{+}\right) 352.0119$, found 352.0113. 
3-((2,4-Dibromophenyl)carbamoyl)-7-oxabicyclo[2.2.1]heptane-2-carboxylic acid (II-27). Yield, 18\%, white solid, mp: $241-242{ }^{\circ} \mathrm{C} ;{ }^{1} \mathrm{H}-\mathrm{NMR}$ 8: 1.59-1.68 (m, 4H, H-1, 2), $3.14(\mathrm{~s}, 2 \mathrm{H}, \mathrm{H}-4,5), 4.79$ (d, J = $4.10 \mathrm{~Hz}$, 1H, H-6), 4.91 (br, s, 1H, H-3), $7.58\left(\mathrm{~d}, J=8.83 \mathrm{~Hz}, 1 \mathrm{H}, \mathrm{H}-5^{\prime}\right), 7.88\left(\mathrm{~s}, 1 \mathrm{H}, \mathrm{H}-6^{\prime}\right), 7.95(\mathrm{~d}, J=8.83 \mathrm{~Hz}, 1 \mathrm{H}$, H-3'), 9.06 (s, 1H, H-9), 12.31 (br, s, 1H, H-10); ${ }^{13}$ C-NMR $8: 28.77$ (1C, C-1), 28.99 (1C, C-2), 52.46 (1C, C-4), 54.78 (1C, C-5), 77.72 (1C, C-3), 79.37 (1C, C-6), 115.70 (1C, C-4' ), 116.51 (1C, C-2'), 125.09 (1C, C-6'), 131.40 (1C, C-3'), 134.66 (1C, C-5'), 136.36 (1C, C-1'), 170.37 (1C, C-8), 172.56 (1C, C-7); HR-MS (ESI): $m / z$ calcd for $\mathrm{C}_{14} \mathrm{H}_{13} \mathrm{Br}_{2} \mathrm{NO}_{4} \mathrm{Na}\left([\mathrm{M}+\mathrm{Na}]^{+}\right) 441.9089$, found 441.9096 .

3-((4-Iodo-2-methylphenyl)carbamoyl)-7-oxabicyclo[2.2.1]heptane-2-carboxylic acid (II-28). Yield, 56\%, white solid, mp: $147-149{ }^{\circ} \mathrm{C}$; ${ }^{1} \mathrm{H}-\mathrm{NMR} \delta: 1.59$ (br, s, 4H, H-1, 2), 2.26 (s, 3H, H-7'), 3.04-3.10 (m, $1 \mathrm{H}, \mathrm{H}-4), 3.12-3.18(\mathrm{~m}, 1 \mathrm{H}, \mathrm{H}-5), 4.74-4.79(\mathrm{~m}, 1 \mathrm{H}, \mathrm{H}-6), 4.81-4.87(\mathrm{~m}, 1 \mathrm{H}, \mathrm{H}-3), 7.42\left(\mathrm{~s}, 1 \mathrm{H}, \mathrm{H}-5^{\prime}\right)$, $7.67\left(\mathrm{~d}, J=8.20 \mathrm{~Hz}, 1 \mathrm{H}, \mathrm{H}-6^{\prime}\right), 7.80\left(\mathrm{~d}, J=7.88 \mathrm{~Hz}, 1 \mathrm{H}, \mathrm{H}-4^{\prime}\right), 9.29-9.35(\mathrm{~m}, 1 \mathrm{H}, \mathrm{H}-9), 12.17-12.21(\mathrm{~m}$, 1H, H-10); ${ }^{13}$ C-NMR 8: 13.76 (1C, C-7'), 28.91 (1C, C-1), 29.22 (1C, C-2), 52.33 (1C, C-4), 53.72 (1C, C-5), 77.57 (1C, C-3), 79.13 (1C, C-6), 120.49 (1C, C-4'), 125.27 (1C, C-6' $), 126.95$ (1C, C-2'), 129.20 (1C, C-5'), 128.61 (1C, C-1'), 151.26 (1C, C-3'), 170.18 (1C, C-8), 172.59 (1C,C-7); HR-MS (ESI): $m / z$ calcd for $\mathrm{C}_{15} \mathrm{H}_{16} \mathrm{INO}_{4} \mathrm{Na}\left([\mathrm{M}+\mathrm{Na}]^{+}\right) 424.0022$, found 424.0025 .

3-((3,5-Dimethoxyphenyl)carbamoyl)-7-oxabicyclo[2.2.1]heptane-2-carboxylic acid (II-29). Yield, 74\%, white solid, mp: $156-157^{\circ} \mathrm{C} ;{ }^{1} \mathrm{H}-\mathrm{NMR}$ 8: $1.47-1.68(\mathrm{~m}, 4 \mathrm{H}, \mathrm{H}-1,2), 2.94(\mathrm{~d}, J=9.77 \mathrm{~Hz}, 1 \mathrm{H}, \mathrm{H}-4), 3.05$ (d, $J=9.46 \mathrm{~Hz}, 1 \mathrm{H}, \mathrm{H}-5), 3.71\left(\mathrm{~s}, 6 \mathrm{H}, \mathrm{H}-7^{\prime}, 8^{\prime}\right), 4.63(\mathrm{~d}, J=3.47 \mathrm{~Hz}, 1 \mathrm{H}, \mathrm{H}-6), 4.79$ (br, s, 1H, H-3), 6.20

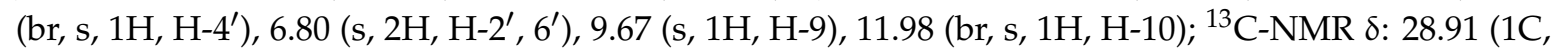
C-1), 29.42 (1C, C-2), 40.02 (2C, C-7', C-8' ), 52.33 (1C, C-4), 53.89 (1C, C-5), 77.49 (1C, C-3), 78.97 (1C, C-6), 105.05 (1C, C-4' $), 119.56$ (2C, C-2',$\left.C-6^{\prime}\right), 133.65$ (1C, C-1'), 143.99 (2C, C-3',$\left.C-5^{\prime}\right), 170.62$ (1C, C-8), 172.57 (1C, C-7); HR-MS (ESI): $m / z$ calcd for $\mathrm{C}_{16} \mathrm{H}_{19} \mathrm{NO}_{6} \mathrm{Na}\left([\mathrm{M}+\mathrm{Na}]^{+}\right)$344.1110, found 344.1111.

3-((2,5-Dimethoxyphenyl)carbamoyl)-7-oxabicyclo[2.2.1]heptane-2-carboxylic acid (II-30). Yield 64\%, white solid, mp: $188-189^{\circ} \mathrm{C} ;{ }^{1} \mathrm{H}-\mathrm{NMR} \delta: 1.58$ (br, s, 4H, H-1, 2), 2.97-3.23 (m, 2H, H-4, 5), 3.55-3.94 (m, 6H, H-7', $8^{\prime}$ ), 4.71 (br, s, 1H, H-6), 4.89 (br, s, 1H, H-3), 6.58 (br, s, 1H, H-4'), 6.94 (br, s, 1H, H-3'), 7.83 (br, s, 1H, H-6'), 8.89 (br, s, 1H, H-9), 12.10-12.40 (m, 1H, H-10); ${ }^{13} \mathrm{C}-\mathrm{NMR}$ 8: 28.8 (1C, C-1), 28.96 (1C, C-2), 52.46 (1C, C-4), 55.33 (1C, C-5), 55.80 (1C, C-8'), 57.11 (1C, C-7'), 77.57 (1C, C-3), 79.50 (1C, C-6), 107.14 $\left(1 \mathrm{C}, \mathrm{C}-6^{\prime}\right), 107.58\left(1 \mathrm{C}, \mathrm{C}-3^{\prime}\right), 112.29\left(1 \mathrm{C}, \mathrm{C}-4^{\prime}\right), 129.20\left(1 \mathrm{C}, \mathrm{C}-1^{\prime}\right), 143.25\left(1 \mathrm{C}, \mathrm{C}-2^{\prime}\right), 153.64\left(1 \mathrm{C}, \mathrm{C}-5^{\prime}\right)$, 170.11 (1C, C-8), 172.59 (1C, C-7); HR-MS (ESI): $m / z$ calcd for $\mathrm{C}_{16} \mathrm{H}_{19} \mathrm{NO}_{6} \mathrm{Na}\left([\mathrm{M}+\mathrm{Na}]^{+}\right)$344.1110, found 344.1119 .

3-((2-Methyl-3-nitrophenyl)carbamoyl)-7-oxabicyclo[2.2.1]heptane-2-carboxylic acid (II-31). Yield, 35\%, white solid, mp: $144-145{ }^{\circ} \mathrm{C} ;{ }^{1} \mathrm{H}-\mathrm{NMR} \delta: 1.59$ (br, s, 4H, H-1, 2), 2.26 (s, 3H, H-7'), 3.04-3.10 (m, 1H, H-4), 3.12-3.18 (m, 1H, H-5), 4.74-4.79 (m, 1H, H-6), 4.81-4.87 (m, 1H, H-3), 7.42 (s, 1H, H-5'), $7.67\left(\mathrm{~d}, J=8.20 \mathrm{~Hz}, 1 \mathrm{H}, \mathrm{H}-6^{\prime}\right), 7.80\left(\mathrm{~d}, J=7.88 \mathrm{~Hz}, 1 \mathrm{H}, \mathrm{H}-4^{\prime}\right), 9.29-9.35$ (m, 1H, H-9), 12.17-12.21 (m,

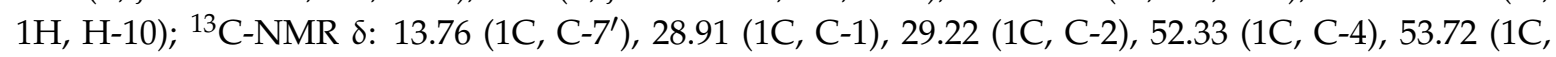
C-5), 77.57 (1C, C-3), 79.13 (1C, C-6), 120.49 (1C, C-4'), 125.27 (1C, C-6' ), 126.95 (1C, C-2' ), 129.20 (1C, C-5'), 128.61 (1C, C-1'), 151.26 (1C, C-3'), 170.18 (1C, C-8), 172.59 (1C,C-7); HR-MS (ESI): $m / z$ calcd for $\mathrm{C}_{15} \mathrm{H}_{16} \mathrm{~N}_{2} \mathrm{O}_{6} \mathrm{Na}\left([\mathrm{M}+\mathrm{Na}]^{+}\right)$343.0906, found 343.0911.

3-(Naphthalen-1-ylcarbamoyl)-7-oxabicyclo[2.2.1]heptane-2-carboxylic acid (II-32). Yield, 69\%, white solid, mp: 187-188 ${ }^{\circ} \mathrm{C} ;{ }^{1} \mathrm{H}-\mathrm{NMR}$ 8: 1.62 (br, s, 4H, H-1, 2), 3.11 (d, J = $\left.9.77 \mathrm{~Hz}, 1 \mathrm{H}, \mathrm{H}-4\right), 3.29$ (s, 1H, H-5), 4.79-4.85 (m, 1H, H-6), 4.86-4.91 (m, 1H, H-3), 7.49 (s, 1H, H-2'), 7.53-7.61 (m, 2H, H-3', 9'), 7.73 (s, $\left.1 \mathrm{H}, \mathrm{H}-8^{\prime}\right), 7.78-7.84\left(\mathrm{~m}, 1 \mathrm{H}, \mathrm{H}-4^{\prime}\right), 7.91-7.96\left(\mathrm{~m}, 1 \mathrm{H}, \mathrm{H}-7^{\prime}\right), 8.03-8.08$ (m, 1H, H-10'), 9.57 (s, 1H, H-9), 12.18 (s, 1H, H-10); ${ }^{13} \mathrm{C}-\mathrm{NMR}$ 8: 28.97 (1C, C-1), 29.28 (1C, C-2), 52.36 (1C, C-4), 54.14 (1C, C-5), 77.60 (1C, C-3), 79.49 (1C, C-6), 120.54 (1C, C-2'), 122.63 (1C, C-4' $), 125.01\left(1 \mathrm{C}, \mathrm{C}-7^{\prime}\right), 125.99$ (1C, C-6' $), 126.28$ $\left(1 \mathrm{C}, \mathrm{C}-8^{\prime}\right), 126.43\left(1 \mathrm{C}, \mathrm{C}-9^{\prime}\right), 127.47\left(1 \mathrm{C}, \mathrm{C}-3^{\prime}\right), 128.61$ (1C, C-10'), 134.04 (1C, C-5'), 134.09 (1C, C-1'), 170.35 (1C, C-8), 172.86 (1C, C-7); HR-MS (ESI): $m / z$ calcd for $\mathrm{C}_{18} \mathrm{H}_{17} \mathrm{NO}_{4} \mathrm{Na}\left([\mathrm{M}+\mathrm{Na}]^{+}\right)$334.1055, found 334.1056 . 
3-(Pyridin-2-ylcarbamoyl)-7-oxabicyclo[2.2.1]heptane-2-carboxylic acid (II-33). Yield, 46\%, white solid, mp: $178-180{ }^{\circ} \mathrm{C} ;{ }^{1} \mathrm{H}-\mathrm{NMR} \delta: 1.46-1.68(\mathrm{~m}, 4 \mathrm{H}, \mathrm{H}-1,2), 3.02(\mathrm{~d}, J=9.46 \mathrm{~Hz}, 1 \mathrm{H}, \mathrm{H}-4), 3.21(\mathrm{~d}, J=9.46 \mathrm{~Hz}$, 1H, H-5), 4.69 (d, J = 5.04 Hz, 1H, H-6), 4.83 (d, J = 3.78 Hz, 1H, H-3), 7.33-7.40 (m, 1H, H-4'), 7.76 $\left(\mathrm{t}, J=7.72 \mathrm{~Hz}, 1 \mathrm{H}, \mathrm{H}-5^{\prime}\right), 7.89\left(\mathrm{~d}, J=4.41 \mathrm{~Hz}, 1 \mathrm{H}, \mathrm{H}-6^{\prime}\right), 8.03\left(\mathrm{~d}, J=8.20 \mathrm{~Hz}, 1 \mathrm{H}, \mathrm{H}-3^{\prime}\right), 9.97(\mathrm{~s}, 1 \mathrm{H}$, H-9); ${ }^{13}$ C-NMR 8: 28.90 (1C, C-1), 29.24 (1C, C-2), 52.28 (1C, C-4), 53.92 (1C, C-5), 77.54 (1C, C-3), 79.28 (1C, C-6), 119.65 (1C, C-6 $), 137.48$ (1C, C-4'), 147.97 (1C, C-5'), 152.43 (1C, C-3'), 160.07 (1C, C-1'), 170.60 (1C, C-8), 172.74 (1C, C-7); HR-MS (ESI): $m / z$ calcd for $\mathrm{C}_{13} \mathrm{H}_{15} \mathrm{~N}_{2} \mathrm{O}_{4}\left([\mathrm{M}+1]^{+}\right) 263.1135$, found 263.1033 .

3-(Thiazol-2-ylcarbamoyl)-7-oxabicyclo[2.2.1]heptane-2-carboxylic acid (II-34). Yield, 56\%, white solid, mp: 174-176 ${ }^{\circ} \mathrm{C} ;{ }^{1} \mathrm{H}-\mathrm{NMR} \delta: 1.42-1.70(\mathrm{~m}, 4 \mathrm{H}, \mathrm{H}-1,2), 3.02(\mathrm{~d}, J=9.46 \mathrm{~Hz}, 1 \mathrm{H}, \mathrm{H}-4), 3.22(\mathrm{~d}, J=9.46 \mathrm{~Hz}$, $1 \mathrm{H}, \mathrm{H}-5), 4.67$ (d, $J=4.10 \mathrm{~Hz}, 1 \mathrm{H}, \mathrm{H}-6), 4.80$ (d, $J=3.15 \mathrm{~Hz}, 1 \mathrm{H}, \mathrm{H}-3), 7.18$ (d, J = 3.47 Hz, 1H, H-5'),

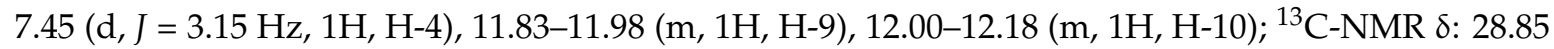
(1C, C-1), 29.42 (1C, C-2), 52.30 (1C, C-4), 52.43 (1C, C-5), 77.61 (1C, C-3), 79.05 (1C, C-6), 113.62 (1C, C-5'), 137.92 (1C, C-4'), 158.54 (1C, C-1'), 169.81 (1C, C-8), 172.45 (1C, C-7); HR-MS (ESI): $m / z$ calcd for $\mathrm{C}_{11} \mathrm{H}_{12} \mathrm{~N}_{2} \mathrm{O}_{4} \mathrm{SNa}\left([\mathrm{M}+\mathrm{Na}]^{+}\right)$291.0415, found 291.0411.

3-(Benzo[d]thiazol-2-ylcarbamoyl)-7-oxabicyclo[2.2.1]heptane-2-carboxylic acid (II-35). Yield, 89\%, white solid, mp: 175-176 ${ }^{\circ} \mathrm{C} ;{ }^{1} \mathrm{H}-\mathrm{NMR}: 1.45-1.73$ (m, 4H, H-1, 2), 3.09 (d, J = 9.14 Hz, 1H, H-4), 3.24 (br, s, 1H, H-5), 4.75 (br, s, 1H, H-6), 4.82 (br, s, 1H, H-3), 7.26-7.47 (m, 2H, H-7' , 8'), 7.70-7.77 (m, 1H, H-6'), 7.94-8.02 (m, 1H, H-9'), 12.19 (br, s, 2H, H-9, 10); ${ }^{13}$ C-NMR 8: 28.87 (1C, C-1), 29.38 (1C, C-2), 52.55 (1C, C-4), 52.63 (1C, C-5), 77.84 (1C, C-3), 78.86 (1C, C-6), 120.84 (1C, C-9'), 122.07 (1C, C-6' ), 123.77 (1C, C-7'), $126.46\left(1 \mathrm{C}, \mathrm{C}-8^{\prime}\right), 131.84\left(1 \mathrm{C}, \mathrm{C}-3^{\prime}\right), 149.01$ (1C, C-4'), $158.52\left(1 \mathrm{C}, \mathrm{C}-1^{\prime}\right), 170.82(1 \mathrm{C}, \mathrm{C}-8)$, 172.31 (1C, C-7); HR-MS (ESI): $m / z$ calcd for $\mathrm{C}_{15} \mathrm{H}_{14} \mathrm{~N}_{2} \mathrm{O}_{4} \mathrm{SNa}\left([\mathrm{M}+\mathrm{Na}]^{+}\right.$) 341.0572, found 341.0579.

3-((1H-Benzo[d]imidazol-2-yl)carbamoyl)-7-oxabicyclo[2.2.1]heptane-2-carboxylic acid (II-36). Yield, 45\%, white solid, mp: $189-190{ }^{\circ} \mathrm{C} ;{ }^{1} \mathrm{H}-\mathrm{NMR} \delta: 1.42-1.70(\mathrm{~m}, 4 \mathrm{H}, \mathrm{H}-1,2), 3.02(\mathrm{~d}, J=9.46 \mathrm{~Hz}, 1 \mathrm{H}, \mathrm{H}-4)$, $3.22(\mathrm{~d}, J=9.46 \mathrm{~Hz}, 1 \mathrm{H}, \mathrm{H}-5), 4.67(\mathrm{~d}, J=4.10 \mathrm{~Hz}, 1 \mathrm{H}, \mathrm{H}-6), 4.80(\mathrm{~d}, J=3.15 \mathrm{~Hz}, 1 \mathrm{H}, \mathrm{H}-3), 7.28$ (dd, $\left.J=5.36,2.84 \mathrm{~Hz}, 2 \mathrm{H}, \mathrm{H}-6^{\prime}, 9^{\prime}\right), 7.61$ (br, s, 2H, H-7' , $\left.8^{\prime}\right), 10.15$ (s, 1H, H-9), 12.05 (s, 1H, H-10); ${ }^{13} \mathrm{C}-\mathrm{NMR}$

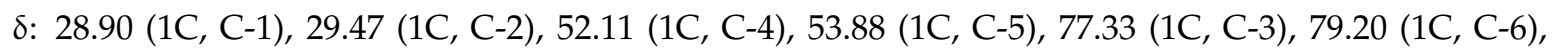
$111.90\left(2 \mathrm{C}, \mathrm{C}-6^{\prime}, \mathrm{C}^{-9^{\prime}}\right), 119.73\left(2 \mathrm{C}, \mathrm{C}-7^{\prime}, \mathrm{C}-8^{\prime}\right), 138.96$ (2C, C-3', C-4'), $155.36\left(1 \mathrm{C}, \mathrm{C}-1^{\prime}\right), 176.17$ (2C, C-7, C-8); HR-MS (ESI): $m / z$ calcd for $\mathrm{C}_{15} \mathrm{H}_{15} \mathrm{~N}_{3} \mathrm{O}_{4} \mathrm{Na}\left([\mathrm{M}+\mathrm{Na}]^{+}\right.$) 324.0960, found 324.0963.

\subsection{Screening of Antifungal Activity in Vitro}

The antifungal activity of the synthetic compounds in vitro against eight plant pathogenic fungi (Valsa mali, B. berengeriana, S. fructigena, G. cingulate, A. alternate, S. sclerotiorum, A. solani and C. sativum) was assayed by the mycelium growth rate method with slight modification [27]. All of the fungi were provided by Laboratory of Integrated Management of Plant Diseases, Northwest A \& F University (Yangling, China). The isolates were cultured for 5 days at $25 \pm 1{ }^{\circ} \mathrm{C}$ on potato dextrose agar (PDA).

Antifungal activity was assessed as follows: the synthesized compounds were screened in vitro for their antifungal activities against the eight phytopathogenic fungi. PDA medium was prepared in the flasks and sterilized. Those compounds were dissolved in DMSO at a concentration of $50 \mu \mathrm{g} / \mathrm{mL}$. DMSO served as the control, while commercially available agricultural fungicide thiabendazole was used as a positive control for its high efficiency and broad spectrum of antifungal activity. Each sample was measured in three replicates, each colony diameter of the three replicates was measured four times by a cross bracketing method. After the mycelia completed growth, the diameters of the mycelial masses were measured and the inhibition rates were calculated according to the following formula and expressed as means \pm S.D.:

$$
\text { Growth inhibition rate }(\%)=\left[\left(d_{c}-d_{0}\right)-\left(d_{t}-d_{0}\right)\right] /\left(d_{c}-d_{0}\right) \times 100
$$


where $d_{0}$ : diameter of the fungus cut-outs, $d_{c}$ : average diameter of the untreated control fungus, and $d_{t}$ is the average diameter of mycelia on treated PDA with those compounds.

Based on the results of preliminary screening, the final stock solutions of the tested compounds dissolved in acetone were 100, 50, 25, 10, and $5 \mu \mathrm{g} / \mathrm{mL}$. The medium was then poured into sterilized Petri dishes. All types of fungi were incubated in PDA at $25 \pm 1{ }^{\circ} \mathrm{C}$ for 5 days to get new mycelium for the antifungal assays, and a mycelia disk of approximately $5 \mathrm{~mm}$ diameter cut-out from culture medium was picked up with a sterilized inoculation needle and inoculated in the center of the PDA Petri dishes with different concentrations of NCTD derivatives [29,30]. The inoculated Petri dishes were incubated at $25 \pm 1{ }^{\circ} \mathrm{C}$ for 4 days. The $\mathrm{IC}_{50}$ (median inhibitory concentration) values of some compounds were determined, and the results are listed in Table 2.

\section{Conclusions}

In summary, we have reported the synthesis of a series of NCTD derivatives with aromatic amine moieties as well as the ability of these compounds to inhibit the growth of eight fungal phytopathogens. Seven of these synthetic compounds presented significant fungistatic activities against all of the eight fungi, superior to the corresponding parent compound NCTD for some fungi, and in some cases they were the same as or more active than TBZ. Compound II-8 exhibited the most significant activity on all eight fungi, much better than TBZ, NCTD and CTD. In particular, II-8 showed excellent antifungal properties against $S$. fructigena and S. sclerotiorum, with $\mathrm{IC}_{50}$ values of 0.88 and $0.97 \mu \mathrm{g} / \mathrm{mL}$, respectively. SAR data for these compounds are as follows: (1) the benzene ring is critical for the improvement of the spectrum of antifungal activity and the inhibition of B. berengeriana, G. cingulate, A. alternate, S. sclerotiorum, A. solani and C. sativum (c.f. II-1 vs. norcantharidin and cantharidin); (2) among the three sites, including the $\mathrm{C}-2^{\prime}, \mathrm{C}-3^{\prime}$ and $\mathrm{C}-4^{\prime}$ positions of the phenyl ring, the presence of the halogen atom at the $\mathrm{C}-3^{\prime}$ position of the phenyl ring caused the most significant increase in antifungal activity (II-5 vs. II-4 and II-6, II-8 vs. II-7 and II-9, II-11 vs. II-10 and II-12); (3) compounds with substitutions of strongly electron-drawing or electron-donating groups were found to have a poor antifungal activity; and (4) compared with fluorine, bromine and iodine, one chlorine atom substituted at $\mathrm{C}-3^{\prime}$ position of the benzene ring gave the highest fungistatic activity (II-8 vs. II-5, II-11 and II-14). Taken together, the data demonstrated that compound II-8 possesses the most potent inhibitory activity toward the fungal plant pathogens tested in this study and could be a potential lead structure for further discovery of novel antifungal agrochemicals.

Acknowledgments: We sincerely appreciate John Richard Schrock (Emporia State University, Kansas, USA) and Sang-Youl Park (University of California, Riverside, CA, USA) for revising the manuscript. This research is supported by the Special Fund for the Public Interest (Agriculture) (200903052) by The Ministry of Science and Technology and The Ministry of Agriculture of China, the Shaanxi Agriculture Science and Technology Projects (2014K01-25-02) and The Ministry of Agriculture of China and the "13115" Sci-Tech Innovation Project of Shaanxi Province (2007ZDKG-14).

Author Contributions: W.S. and Y.W. conceived and designed the experiments; W.S., S.Z. and H.W. performed the experiments; W.S. and S.Z. analyzed the data; Y.Z. contributed reagents/materials/analysis tools; W.S. and Y.W. wrote the paper.

Conflicts of Interest: The authors declare no conflict of interest.

\section{References}

1. Bai, Y.B.; Zhang, A.L.; Tang, J.J.; Gao, J.M. Synthesis and antifungal activity of 2-chloromethyl-1Hbenzimidazole derivatives against phytopathogenic fungi in vitro. J. Agric. Food Chem. 2013, 61, 2789-2795. [CrossRef] [PubMed]

2. Schirra, M.; Aquino, S.D.; Cabras, P.; Angioni, A. Control of postharvest diseases of fruit by heat and fungicides: Efficacy, residue levels, and residue persistence. A review. J. Agric. Food Chem. 2011, 59, 8531-8542. [CrossRef] [PubMed]

3. Savary, S.; Teng, P.S.; Willocquet, L.; Nutter, F.W., Jr. Quantification and modeling of crop losses: A review of purposes. Annu. Rev. Phytopathol. 2006, 44, 89-112. [CrossRef] [PubMed] 
4. Zhang, Y.J.; Yu, J.J.; Zhang, Y.N.; Zhang, X.; Cheng, C.J.; Wang, J.X.; Hollomon, D.W.; Fan, P.S.; Zhou, M.G. Effect of carbendazim resistance on trichothecene production and aggressiveness of Fusarium graminearum. Mol. Plant Microbe Interact. 2009, 22, 1143-1150. [CrossRef] [PubMed]

5. Seifi, A.; Visser, R.G.F.; Bai, Y.L. How to effectively deploy plant resistances to pests and pathogens in crop breeding. Euphytica 2013, 190, 321-334. [CrossRef]

6. Dossey, A.T. Insects and their chemical weaponry: New potential for drug discovery. Nat. Prod. Rep. 2010, 27, 1737-1757. [CrossRef] [PubMed]

7. Zhu, J.W.J.; Li, A.Y.; Pritchard, S.; Tangtrakulwanich, K.; Baxendale, F.P.; Brewer, G. Contact and Fumigant Toxicity of a Botanical-Based Feeding Deterrent of the Stable Fly, Stomoxys calcitrans (Diptera: Muscidae). J. Agric. Food Chem. 2011, 59, 10394-10400. [CrossRef] [PubMed]

8. Dayan, F.E.; Cantrell, C.L.; Duke, S.O. Natural products in crop protection. Bioorg. Med. Chem. 2009, 17, 4022-4034. [CrossRef] [PubMed]

9. Nicholls, L.C.; Teare, D. Poisoning by cantharides. Br. J. Med. 1954, 2, 1384-1386. [CrossRef]

10. Wang, G.S. Medical uses of Mylabris in ancient China and recent studies. J. Ethnopharmacol. 1989, 26, 147-162. [CrossRef]

11. Sakoff, J.A.; Ackland, S.P.; Baldwin, M.L.; Keane, M.A.; McCluskey, A. Anticancer activity and protein phosphatase 1 and 2A inhibition of a new generation of cantharidin analogues. Investig. New Drugs 2002, 20, 1-11. [CrossRef]

12. Li, W.; Xie, L.; Chen, Z.; Zhu, Y.; Sun, Y.J.; Miao, Y.; Xu, Z.K.; Han, X. Cantharidin, a potent and selective PP2A inhibitor, induces an oxidative stress independent growth inhibition of pancreatic cancer cells through G2/M cell-cycle arrest and apoptosis. Cancer Sci. 2010, 101, 1226-1233. [CrossRef] [PubMed]

13. McCluskey, A.; Ackland, S.P.; Bowyer, M.C.; Baldwin, M.L.; Garner, J.; Walkom, C.C.; Sakoff, J.A. Cantharidin analogues: Synthesis and evaluation of growth inhibition in a panel of selected tumour cell lines. Bioorg. Chem. 2003, 31, 68-79. [CrossRef]

14. Sagawa, M.; Nakazato, T.; Uchida, H.; Ikeda, Y.; Kizaki, M. Cantharidin induces apoptosis of human multiple myeloma cells via inhibition of the JAK/STAT pathway. Cancer Sci. 2008, 99, 1820-1826. [CrossRef] [PubMed]

15. Carrel, J.E.; Eisner, T. Cantharidin potent feeding deterrent to insects. Science 1974, 183, 755-757. [CrossRef] [PubMed]

16. Carrel, J.E.; Doom, J.P.; McCormick, J.P. Identification of cantharidin in false blister beetles (Coleoptera. Meloidae) from Florida. J. Chem. Ecol. 1986, 12, 741-747. [CrossRef] [PubMed]

17. Li, X.F.; Chen, X.S.; Hou, X.H. The toxicity effect of cantharidin on six pests. Guizhou Agric. Sci. 2008, 36, 65-66.

18. Liu, R.R.; Ma, Y.; Ma, Z.Q.; Zhang, Y.L. Bioactivity of cantharidin against eleven pests. J. Northwest A F Univ. 2010, 38, 181-185.

19. Bajsa, J.; Duke, S.O.; Tekwani, B.L. Plasmodium falciparum serine/threonine phosphoprotein phosphatases (PPP): From housekeeper to the "Holy Grail". Curr. Drug Targets 2008, 9, 997-1012. [CrossRef] [PubMed]

20. Lin, X.H.; Walter, J.; Scheidmann, K.; Ohst, K.; Newport, J.; Walter, G. Protein phosphatase 2A is required for the initiation of chromosomal DNA replication. Proc. Natl. Acad. Sci. USA 1998, 95, 14693-14698. [CrossRef] [PubMed]

21. Moorhead, G.B.; de Wever, V.; Templeton, G.; Kerk, D. Evolution of protein phosphatases in plants and Animals. Biochem. J. 2009, 417, 401-409. [CrossRef] [PubMed]

22. Bertini, I.; Calderone, V.; Fragai, M.; Luchinat, C.; Talluri, E. Structural basis of serine/threonine phosphatase inhibition by the archetypal small molecules cantharidin and norcantharidin. J. Med. Chem. 2009, 52, 4838-4843. [CrossRef] [PubMed]

23. Li, Y.M.; Mackintosh, C.; Casida, J.E. Protein phosphatase $2 \mathrm{~A}$ and its $\left[{ }^{3} \mathrm{H}\right]$ cantharidin $/\left[{ }^{3} \mathrm{H}\right]$ endothall thioanhydride binding site. Inhibitor specificity of cantharidin and ATP analogues. Biochem. Pharmacol. 1993, 46, 1435-1443. [CrossRef]

24. Chen, X.E.; Liu, J.Y.; Zhang, Y.L. Cantharidin impedes the activity of protein serine/threonine phosphatase in Plutella xylostella. Mol. BioSyst. 2014, 10, 240-250. [CrossRef] [PubMed]

25. Bajsa, J.; Pan, Z.Q.; Dayan, F.E.; Owens, D.K.; Duke, S.O. Validation of serine/threonine protein phosphatase as the herbicide target site of endothall. Pestic. Biochem. Physiol. 2012, 102, 38-44. [CrossRef] 
26. Sun, W.B.; Liu, Z.Y.; Zhang, Y.L. Cantharidin and its anhydride-modified derivatives: Relation of structure to insecticidal activity. Int. J. Mol. Sci. 2013, 14, 1-16. [CrossRef] [PubMed]

27. Li, X.J.; Zhang, Q.; Zhang, A.L.; Gao, J.M. Metabolites from Aspergillus fumigatus, an endophytic fungus associated with Melia azedarach, and their antifungal, antifeedant, and toxic activities. J. Agric. Food Chem. 2012, 60, 3424-3431. [CrossRef] [PubMed]

28. Hill, T.A.; Stewart, S.G.; Gordon, C.P.; Ackland, S.P.; Gilbert, J.; Sauer, B.; Sakoff, J.A.; McCluskey, A. Norcantharidin Analogues: Synthesis, Anticancer Activity and Protein Phosphatase 1 and 2A Inhibition. ChemMedChem 2008, 3, 1878-1892. [CrossRef] [PubMed]

29. Saremi, H.; Okhovvat, S.M. Mycotoxin producing Fusarium species associated with plant disease on potato, wheat, corn and animal diseases in northwest Iran. Commun. Agric. Appl. Biol. Sci. 2006, 71, 1175-1185. [PubMed]

30. Popov, I.I.; Narezhnaya, V.N.; Zubenko, A.A. Research on unsaturated azole derivatives VII. New syntheses in the 2-vinylbenzimidazole series. Khim. Geterotsikl. Soedin. 1978, 8, 1104-1107.

Sample Availability: Samples of the compounds II (1-36) are available from the authors.

(C) 2015 by the authors; licensee MDPI, Basel, Switzerland. This article is an open access article distributed under the terms and conditions of the Creative Commons by Attribution (CC-BY) license (http://creativecommons.org/licenses/by/4.0/). 